ARTICLE

Received 12 Mar 2014 | Accepted 16 Jun 2014 | Published 15 Jul $2014 \quad$ DOl: 10.1038/ncomms5407

\title{
Rab8a interacts directly with PI3K $\gamma$ to modulate TLR4-driven PI3K and mTOR signalling
}

Lin Luo ${ }^{1, \star}$, Adam A. Wall ${ }^{1, \star}$, Jeremy C. Yeo ${ }^{1}$, Nicholas D. Condon ${ }^{1}$, Suzanne J. Norwood, Simone Schoenwaelder ${ }^{2,3}$, Kaiwen W. Chen ${ }^{1}$, Shaun Jackson ${ }^{2,3}$, Brendan J. Jenkins ${ }^{4}$, Elizabeth L. Hartland ${ }^{5}$, Kate Schroder ${ }^{1}$, Brett M. Collins ${ }^{1}$, Matthew J. Sweet ${ }^{1} \&$ Jennifer L. Stow ${ }^{1}$

Toll-like receptor 4 (TLR4) is activated by bacterial lipopolysaccharide (LPS) to mount innate immune responses. The TLR4-induced release of pro- and anti-inflammatory cytokines generates robust inflammatory responses, which must then be restrained to avoid disease. New mechanisms for the critical regulation of TLR-induced cytokine responses are still emerging. Here we find TLR4 complexes localized in LPS-induced dorsal ruffles on the surface of macrophages. We discover that the small GTPase Rab8a is enriched in these ruffles and recruits phosphatidylinositol 3-kinase (PI3K $\gamma$ ) as an effector by interacting directly through its Ras-binding domain. Rab8a and PI3K $\gamma$ function to regulate Akt signalling generated by surface TLR4. Rab8a and PI3K $\gamma$ do not affect TLR4 endocytosis, but instead regulate mammalian target of rapamycin signalling as a mechanism for biasing the cytokine profile to constrain inflammation in innate immunity.

\footnotetext{
${ }^{1}$ Institute for Molecular Bioscience, The University of Queensland, Brisbane, Queensland 4072, Australia. ${ }^{2}$ Australian Centre for Blood Diseases, Monash University, Melbourne, Victoria 3004, Australia. ${ }^{3}$ Heart Research Institute \& Charles Perkins Centre, The University of Sydney, Sydney, New South Wales 2006, Australia. ${ }^{4}$ Centre for Innate Immunity and Infectious Diseases, MIMR-PHI Institute of Medical Research, Monash University, $27-31$ Wright Street, Clayton, Victoria 3168, Australia. ${ }^{5}$ Department of Microbiology and Immunology, University of Melbourne at the Peter Doherty Institute for Infection and Immunity, Melbourne, Victoria 3010, Australia. ${ }^{\star}$ These authors contributed equally to the work. Correspondence and requests for materials should be addressed to J.L.S. (email: j.stow@imb.uq.edu.au).
} 
$\mathrm{M}$ acrophages are key sentinels of the innate immune system, tasked with detecting and responding to pathogens. Accordingly, the macrophage cell surface is armed with various membrane protrusions, is serviced by multiple endocytic and exocytic pathways and has a high rate of plasma membrane turnover to accommodate environmental sampling and pathogen sensing ${ }^{1}$. Members of the Rab family of small GTPases regulate multiple steps of membrane trafficking ${ }^{2}$ and, in macrophages, Rabs associated with the cell surface and endocytic pathways are uniquely poised to influence pathogen recognition and internalization ${ }^{3-5}$. Multiple Rabs are involved in the phagocytosis of pathogens $s^{6,7}$ and in the trafficking and secretion of cytokines in pathogen-activated cells ${ }^{1}$. Several $\mathrm{Rabs}^{3-5}$ have also been implicated in regulating the trafficking of Toll-like receptor 4 (TLR4), a member of the TLR family of pattern recognition receptors that initiate cellular responses to pathogens and danger signals ${ }^{8}$.

TLR4 recognizes the lipopolysaccharide (LPS) component of Gram-negative bacteria and activates macrophages to initiate responses including the release of inflammatory cytokines and chemokines, production of reactive oxygen species and modulation of cell survival and proliferation ${ }^{9}$. The spatiotemporal regulation of TLR4 and its signalling from either cell surface or endosomal complexes regulates the net outcome of these responses ${ }^{10-12}$. At the cell surface, LPS-bound TLR4 dimerizes for the phosphoinositide-dependent recruitment of its adaptors, Mal (TIRAP) and MyD88 (ref. 13), resulting in the activation of transcription factors, such as NFKB and AP-1, for the production of inflammatory cytokines ${ }^{14,15}$. Surface activation of TLR4 also invokes the phosphatidylinositol 3-kinase (PI3K)/Akt pathway ${ }^{16}$. Inhibiting PI3Ks biases TLR-induced cytokine responses towards a pro-inflammatory setting. Reports have suggested two mechanisms to explain this effect: the regulation of TLR4 endocytosis ${ }^{10}$ and the regulation of downstream TSC/mammalian target of rapamycin (mTOR) signalling ${ }^{16}$. mTOR, a serine/ threonine protein kinase, is the catalytic subunit for both the mTORC1 and mTORC2 complexes ${ }^{17}$. Rapamycin targets the mTORC1 complex downstream of Akt, and during TLR4 signalling, it selectively increases the production of proinflammatory cytokines ${ }^{16}$. Collectively, these complex signalling pathways regulate the inflammatory response, the programming of which is critical for correctly responding to different pathogens and for delimiting inflammation. Dysregulated or excessive cytokine secretion has serious or even fatal consequences in acute conditions like sepsis, and in chronic inflammatory or autoimmune diseases ${ }^{18}$. Importantly, there are multiple regulatory checkpoints that exert tight control of the TLR4 signalling response; however, the full extent and nature of the mechanisms underlying this control remain unclear.

First contact with pathogens such as bacteria is initiated at the cell surface, thus we set out to characterize some of the relevant membrane domains and cellular machinery involved in macrophage activation by LPS. Dorsal ruffles are strikingly upright, and often curved or circular protrusions that accompany pathogen contact and precede macropinocytosis and phagocytosis in activated macrophages ${ }^{19,20}$. In other cell types, dorsal ruffles house growth factor receptors and are sites for initiating growth factor-induced signalling ${ }^{21,22}$. However, dorsal ruffles have received little previous attention with respect to LPS activation of TLR4 (ref. 19). Screening of Rab GTPases for ruffle localization in macrophages drew our attention to Rab8a, a multi-tasking Rab previously localized to leading edges of the cell surface and endo/exocytic pathways in multiple cell types ${ }^{23}$. Here we show that Rab8a and components of the TLR4 complex are enriched in dorsal ruffles where they contribute to LPS-initiated signalling. By mass spectrometry and biochemical analysis, we identified the p110 $\gamma$ catalytic subunit of PI3K as a new effector for Rab8a and thus present, for the first time, a Rab GTPase that binds directly to a class IB PI3K. Furthermore, we show a function for this complex in innate immune signalling. During TLR4 receptor activation, Rab8a and $\mathrm{PI} 3 \mathrm{~K} \gamma$ are together required to fully activate the Akt/mTOR pathway and bias the cytokine response away from a hyperinflammatory setting by decreasing the production of pro-inflammatory cytokines and increasing the production of anti-inflammatory cytokines. Rab8a and $\mathrm{PI} 3 \mathrm{~K} \gamma$ are presented herein as new regulators of signalling in the TLR4 pathway.

\section{Results}

TLR4 and Rab8a in dorsal ruffles of activated macrophages. In macrophages, peripheral Rac-1 and phosphatidic acid dependent ruffling occurs constitutively ${ }^{24}$ and is typically enhanced by growth factors like colony-stimulating factor-1 (ref. 25). Here we show that LPS also increases dorsal ruffling on macrophages, revealing this as an acute macrophage response to contact with Gram-negative bacteria. F-actin-rich dorsal ruffles protruding from LPS-activated cells are increased twofold over those in untreated cells (Fig. 1a and Supplementary Fig. 1a). This mirrors the observation that ruffles do not form after knockdown of TLR4 (ref. 19). Further examination of ruffles in transfected cells revealed that both Citrine-labelled TLR4 and its cell surface adaptor, Mal (as Cerulean-labelled Mal), are enriched in dorsal ruffles (Fig. 1b), denoting these domains as a new location for the pathogen recognition receptor complex of TLR4/Mal/MyD88. Colocalization with Mal places Rab8a in the same ruffles as the TLR4 complex (Fig. 1c). Moreover, under these conditions, Rab8a is actively recruited to the ruffles, rather than being present by passive membrane binding alone. This recruitment can be demonstrated by ratiometric imaging, which highlights the dramatic enrichment of full-length Rab8a protein in dorsal ruffles, compared with the charge-dependent membrane binding of the polybasic Rab8a tail (Fig. 1d and Supplementary Fig. 1b). As established locales for receptor signalling, dorsal ruffles are enriched in signalling phosphoinositides, $\mathrm{PI}(3,4,5) \mathrm{P} 3$ and $\mathrm{PI}(3,4) \mathrm{P} 2$, which can be labelled in live cells with the $\mathrm{PH}$ (pleckstrin homology) domain of Akt (GFP-Akt-PH) ${ }^{26}$. Our LPS-activated cells show strong Akt-PH labelling of Rab8acontaining dorsal ruffles, with both molecules enriched two to threefold, compared with their labelling in other plasma membrane domains (Fig. 1e). This membrane localization of Rab8a is consistent, even at very low expression levels (Supplementary Fig. 2). Based on Rab8a being enriched in LPSinduced dorsal ruffles, we next investigated a possible role for Rab8a in TLR4 signalling.

Rab8a regulates TLR4 signalling and cytokine secretion. To investigate its function, Rab8a was depleted by small hairpin RNA (shRNA) in RAW 264.7 macrophages (to $<30 \%$ of control levels) and cells were treated with LPS to examine downstream signalling responses (Fig. 2). Rab8a-depleted cells show a modest increase in phosphorylation of the MAPK protein, $\mathrm{p} 38$, but no change in ERK1/2 phosphorylation or degradation of the NFKB inhibitory protein, IкB. Notably however, Akt phosphorylation is markedly reduced (by $>60 \%$ at $30 \mathrm{~min}$ ) after depletion of Rab8a (Fig. 2a). To confirm that the altered Akt response could be attributed to Rab8a, we also examined LPS signalling in cell lines stably overexpressing Rab8a (by approximately three to four fold) and found a $\sim 2.5$-fold increase in Akt phosphorylation at 30 and 60 min post LPS (Supplementary Fig. 3a). These results serve to implicate Rab8a in TLR4 signalling and suggest that Rab8a is required for maximal PI3K/Akt activation. 
a

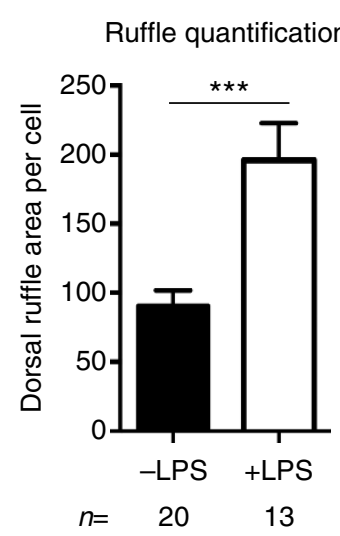

b

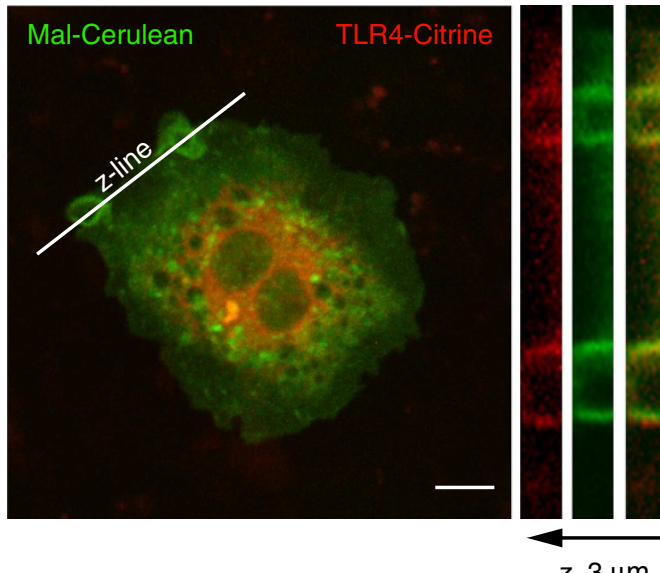

C

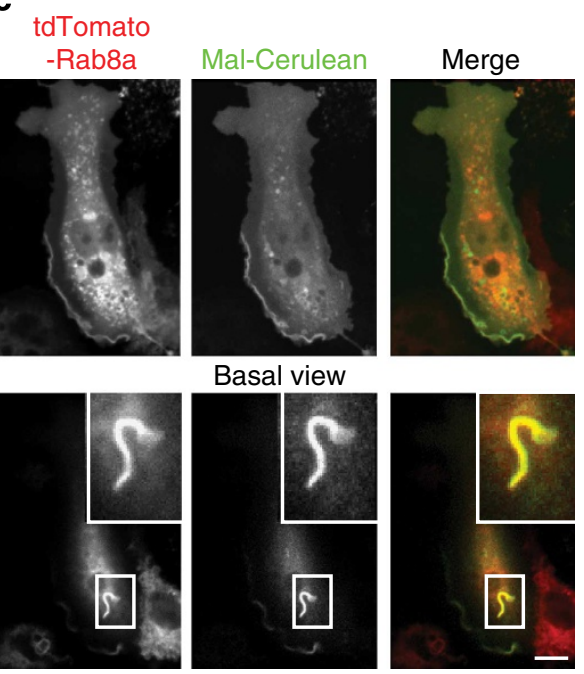

Dorsal view d
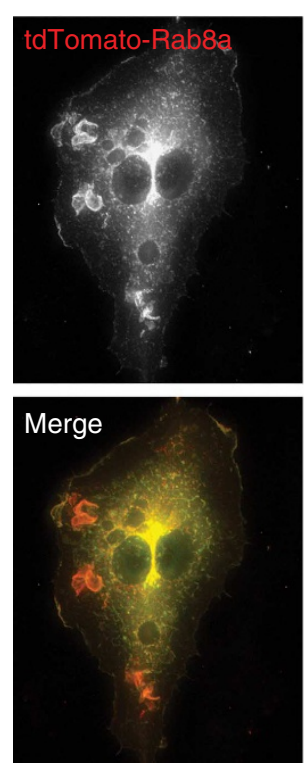

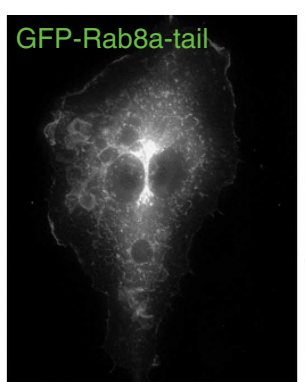

Ratio (Rab8a per tail)

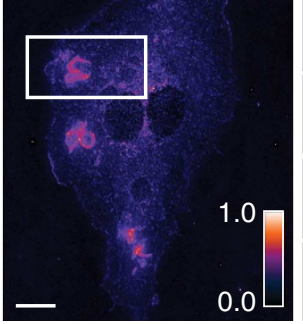

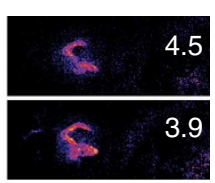
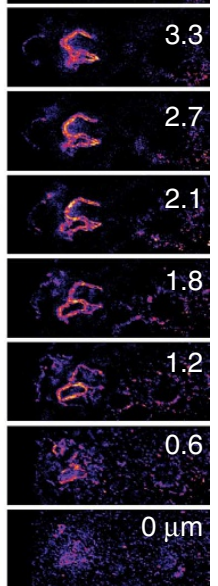

z-slice e
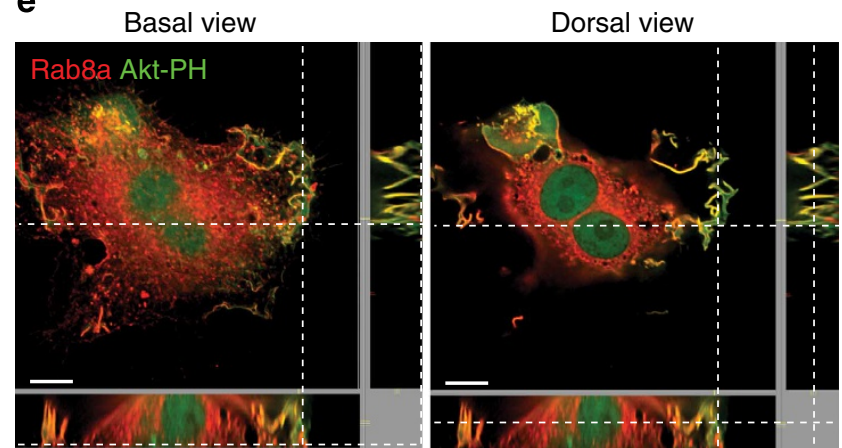

Distribution between

plasma membrane domains

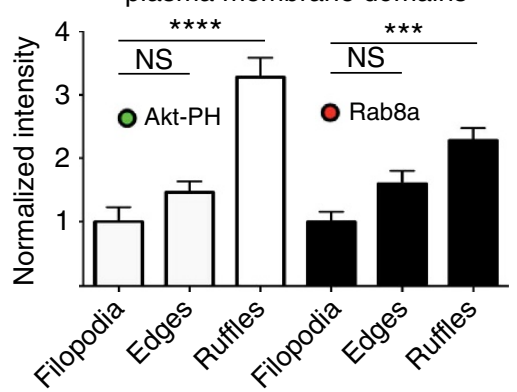

Figure 1 | Rab8a and TLR4 colocalize in LPS-enhanced dorsal ruffles. (a) Quantification of dorsal ruffling on untreated and LPS-treated RAW 264.7 macrophages. Images of ruffling and parameters for ruffle quantification are shown in Supplementary Fig. 1. The data are analysed by Student's $t$-test. ( $n=13-20$ per group) ${ }^{\star \star \star} P<0.001$. (b) A single stack image from live, LPS-treated (15 min) RAW 264.7 cells expressing TLR4-Citrine and Mal-Cerulean shows colocalization at peripheral ruffles. Low TLR4-citrine labelling has been enhanced in the $z$-slice view to visualise cell surface TLR4. Original 16-bit levels (532-18722) enhanced levels (1728-10652). (c) Single $z$-stack (0.19 $\mu \mathrm{m}$ slice) image from live cells transfected with Mal-Cerulean and tdTomato-Rab8a. Upper panel shows first three slices from the base of the cell where Rab8a and Mal colocalize at the cell periphery; lower panel shows the top three slices of the cell where there is colocalization in dorsal ruffles. (d) RAW 264.7 cells coexpressing full-length tdTomato-Rab8a and the GFP-tail domain of Rab8a were used to generate ratiometric images to assess Rab8a enrichment at dorsal ruffles. Inset shows 0.6 $\mu \mathrm{m} z$-slice increments. (e) Single $z$-slice shown in basal and dorsal views where td-Tomato Rab8a and GFP-Akt-PH colocalize in ruffles after 15 min of LPS treatment. Quantification of Rab8a and the Akt-PH probe $(n=5)$ in ruffles and other membrane domains was measured by one-way analysis of variance, ${ }^{\star \star} P<0.01$ and; ${ }^{* \star \star \star} P<0.0001$, respectively. Post-test by Dunnett's multiple comparisons. All images scale bar, $10 \mu \mathrm{m}$.

To test the biological significance of Rab8a depletion, we measured the synthesis and secretion of inflammatory cytokines, which are initiated downstream of LPS-activated TLR4. Over an 8-hour time course, significantly more pro-inflammatory interleukin-6 (IL-6) is produced at both the transcript and secreted levels in Rab8a-depleted cells compared with controls (Fig. 2b). Conversely, the Rab8a-depleted cells display decreased synthesis and secretion of the regulatory cytokine, IL-10 (Fig. 2b). Thus, Rab8a functions in Akt/PI3K signalling to produce a selective or biased cytokine response to TLR4 activation. This is a wholly new 
a

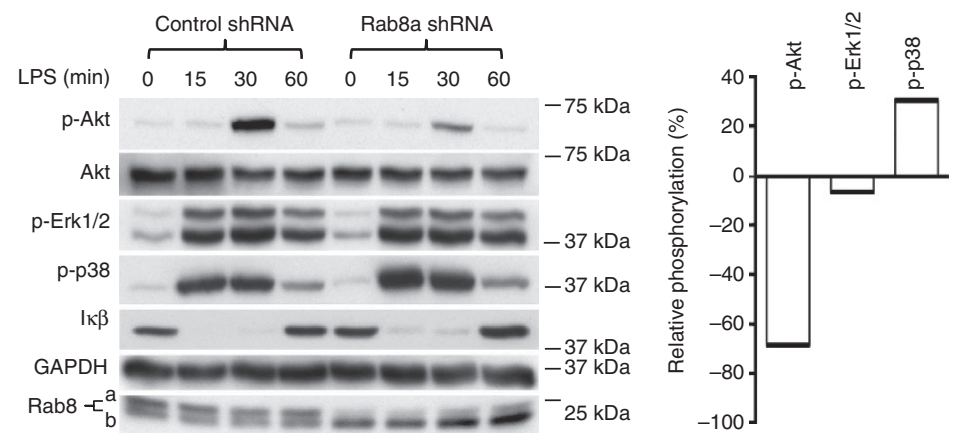

b
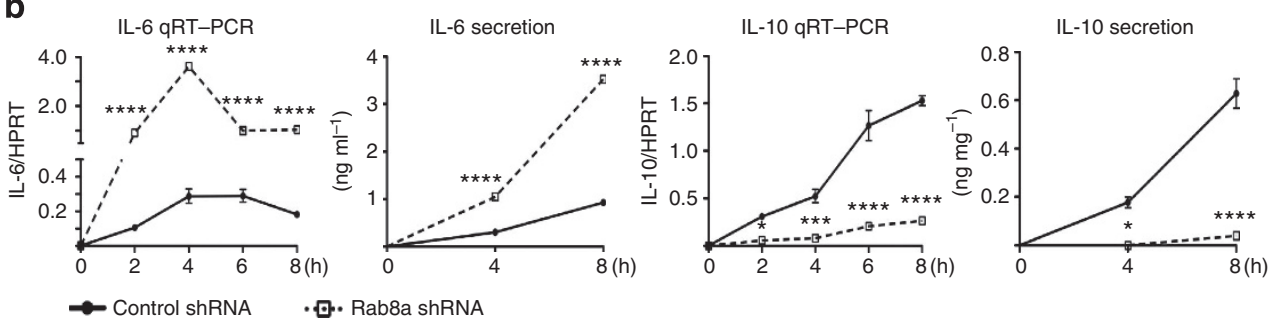

Figure 2 | Rab8 is required for TLR4-mediated signalling. (a) Representative immunoblot analysis of LPS time course for control (scrambled shRNA) and Rab8a-shRNA stable RAW 264.7 cell lines. Phosphorylation of Akt, ERK1/2 and p38 was analysed at 30 min post LPS by densitometry. All immunoblots were cropped for presentation; full-length blots are provided in Supplementary Fig. 5. (b) Control (scrambled shRNA) and Rab8a-shRNA stable RAW 264.7 cell lines were analysed by quantitative reverse transcriptase PCR (qRT-PCR) for transcriptional response and by enzyme-linked immunosorbent assay for the secretion of the cytokines IL- 6 and IL-10. Graphs represent mean \pm s.e.m. ( $n=3$ each). Time course profiles were analysed by two-way analysis of variance (ANOVA). For qRT-PCR; ${ }^{\star \star \star \star} P<0.0001$ for both IL-6 and IL-10. For cytokine secretion ${ }^{\star \star \star \star} P<0.0001$ for IL- 6 and ${ }^{\star \star \star} P<0.001$ for IL-10. Two-way ANOVA were analyzed by Sidak's post-test for multiple comparisons.

role for Rab8a in TLR4 signalling, and one not readily explained by the known Rab8a effectors ${ }^{27}$. We thus set out to identify the relevant effector(s) or binding partners for Rab8a mediating its involvement in TLR4 signalling.

PI3K $\gamma$ is a direct binding partner and effector for Rab8a. To identify binding partners for Rab8a in macrophages, we performed GST-Rab8a pull-down experiments using LPS-activated cell extracts (Fig. 3a). Mass spectrometry analysis revealed a number of known and novel Rab8a-binding partners (Supplementary Table 1a). Among them, a prominent but unexpected binding partner emerged as a band at $120 \mathrm{kDa}$, which was identified convincingly by five independent trypsin-digested peptides, each with $95 \%$ confidence (Fig. 3a,b), as the p110 $\gamma$ catalytic subunit of PI3K (Supplementary Tables 1a,b). Expression of the class 1B $\mathrm{PI} 3 \mathrm{~K} \gamma$ is largely restricted to immune cells where it has been studied in the context of GPCR-dependent neutrophil and T-cell migration ${ }^{28}$, although the highest expression has been reported in myeloid cells, the majority of which are macrophages $^{29}$. To confirm the expression of $\mathrm{PI} 3 \mathrm{~K} \gamma$ in macrophages compared with the other class I PI3Ks $(\alpha, \beta$ and $\delta)$, messenger RNA levels were compared by quantitative reverse transcriptase PCR, and indeed, PI3K $\gamma$ is both immune-specific and is the most abundant isoform in macrophages (Supplementary Fig. 3b). Traditionally for the class $1 \mathrm{~B}$ PI3K, the p110 $\gamma$ catalytic subunit interacts with the p87 or p101 regulatory subunits and with the small GTPase Ras for recruitment to GPCRs ${ }^{30,31}$. The unexpected binding of PI3K $\gamma$ by Rab8a deviates from this model, prompting a more detailed examination to elucidate the nature of this interaction.

Purified proteins produced in bacterial and insect cells were then used for in vitro-binding assays to confirm that Rab8a binds directly to PI3K $\gamma$ (Fig. 3c), but not to other class I PI3K isoforms
(Supplementary Fig. 3c). Furthermore, other Rabs known to function in the TLR4 pathway, such as Rab10 (ref. 3) and $11 \mathrm{a}^{5}$, do not co-immunoprecipitate PI3K $\gamma$ (Supplementary Fig. 3d). This direct interaction is enhanced when Rab8a is in its GTP-bound, active form, as shown by both GTP loading of GST-Rab8a and the co-immunoprecipitation of PI3K $\gamma$ by GFP-Rab8a in the presence of increasing concentrations of a non-hydrolysable GTP analogue, GTP $\gamma \mathrm{S}$ (Fig. 3d,e). These characteristics are consistent with PI3K $\gamma$ interacting with GTP-Rab8a as a new class of Rab effector.

The recruitment of $\mathrm{PI} 3 \mathrm{~K} \gamma$ to membranes is sufficient to initiate its kinase activity, unlike the other class IA PI3K isoforms ${ }^{32}$. In other signalling pathways, this recruitment is mediated by Ras binding directly to the Ras-binding domain (RBD) of PI3 $\mathrm{K}^{30}$. To test whether the RBD is similarly responsible for Rab8a binding, we performed pull-down experiments using the isolated recombinant $\mathrm{RBD}$ from $\mathrm{PI} 3 \mathrm{~K} \gamma$. The $\mathrm{RBD}$ could successfully substitute for full-length protein under the pull-down conditions, confirming that it is necessary and sufficient for binding to Rab8a (Fig. 4a). We then utilized the known crystal structures of GTPbound Rab8 $\mathrm{a}^{33}$ and the co-crystal structure of PI3K $\gamma$ with $\operatorname{Ras}^{34}$ to produce a homology model of Rab8a/PI3K $\gamma$ (Fig. 4b). This model shows a viable alignment of the PI3K $\gamma$ RBD with the effector-binding domain of Rab8a and predicts the critical binding residues on both sides of the interacting surface. Mutation of either F221 or T232 in the RBD or the I41 residues in the effector-binding domain of Rab8a is sufficient to reduce interaction of the two proteins in pull-down experiments (Fig. 4c). These results provide detailed evidence of a direct interaction between Rab8a and PI3K $\gamma$. Rab8a binds to the same $\mathrm{RBD}$ as Ras, and moreover, uses the same critical residues in the $\mathrm{RBD}^{34}$ for its interaction with PI3K $\gamma$. PI3K $\gamma$ normally binds to Ras during its recruitment to activated receptor (GPRC or RTK) complexes. Seeking evidence for such a receptor interaction with 
a

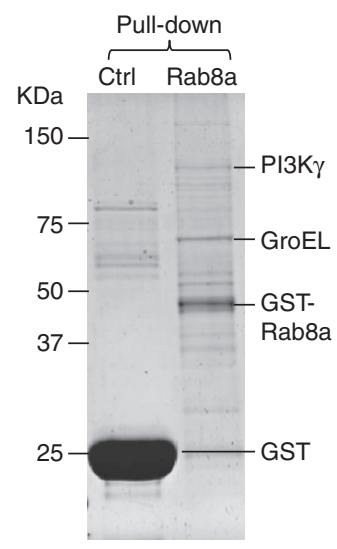

b

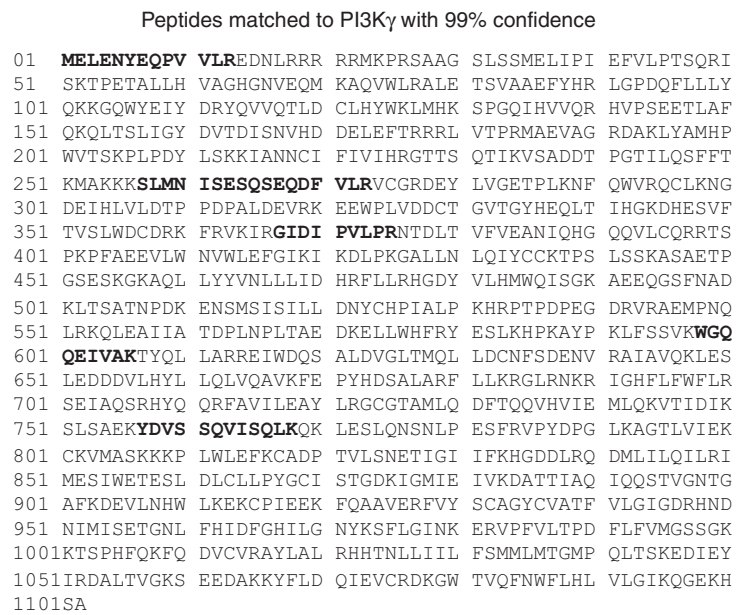

C

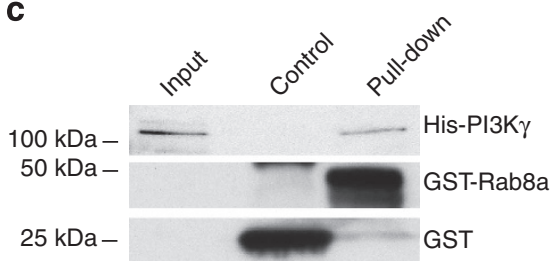

e

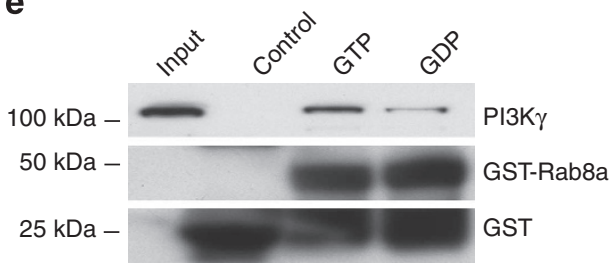

d
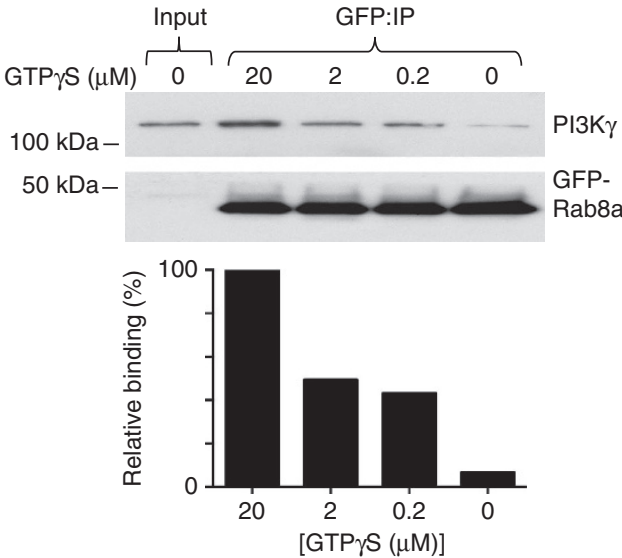

Figure 3 | Identification of PI3K $\boldsymbol{\gamma}$ as a novel effector of Rab8a in macrophages. (a) GST-Rab8a coupled to GSH-Sepharose was used for pull-downs from LPS-activated RAW 264.7 cell extracts. Bound proteins were separated by SDS-PAGE. Excised bands were identified by liquid chromatography/mass spectrometry or mass spectrometry. A major band at $120 \mathrm{kDa}$, absent from the GST control, was identified as PI3K $\gamma$. (b) The sequence coverage of identified peptides in PI3K $\gamma$. Mass spectrometry analysis identified five trypsin-digested peptides from PI3K $\gamma$ with $99 \%$ confidence, including many peptides not found in other PI3K isoforms. (c) Coprecipitation of His-PI3K $\gamma$ by purified GST-Rab8a. (d) Co-immunoprecipitation of PI3K $\gamma$ by GFP-Rab8a in the presence of GTP $\gamma$ S from LPS-treated (15 min) RAW 264.7 cell lysate. Quantification was performed by densitometric analysis of western blots relative to maximal precipitation. (e) Nucleotide exchange of GST-Rab8a with GTP or GDP followed by pull-down of PI3K $\gamma$ from RAW 264.7 cell lysate. All immunoblots were cropped for presentation; full-length blots are provided in Supplementary Fig. 5.

TLR4 revealed that PI3K $\gamma$ does not co-immunoprecipitate with TLR4 (Supplementary Fig. 3e). Taken together, the substitution of Ras with Rab8a and the recruitment of PI3K $\gamma$ independently of TLR4 imply a novel mode for recruitment of PI3K $\gamma$ in the TLR4 pathway.

We then sought to determine the membrane localization of Rab8a and PI3K $\gamma$. Cotransfected macrophages show diffuse cytoplasmic labelling of mCherry-PI3K $\gamma$, typical of PI3K isoforms. In LPS-activated cells, PI3K $\gamma$ is recruited to macrophage ruffles where it colocalizes with GFP-Rab8a (Fig. 4d). Like all class I PI3K isoforms, $\mathrm{PI} 3 \mathrm{~K} \gamma$ converts $\mathrm{PI}(4,5) \mathrm{P} 2$ to $\mathrm{PI}(3,4,5) \mathrm{P} 3$, a function that is in keeping with the phosphoinositide environment of the Rab8positive ruffles (shown in Fig. 1e). Thus we introduce the class IB PI3K $\gamma$ as a novel effector for GTP-Rab8a on ruffle membranes. Next, we further examined this contention by testing whether $\mathrm{PI} 3 \mathrm{~K} \gamma$, like Rab8a, functions in TLR4 signalling.

PI3K $\gamma$ depletion effects on TLR4 signalling and inflammation. As the first of three approaches to explore PI3K $\gamma$ function, we used independent small interfering RNAs targeting PI3K $\gamma$ (Fig. 5a). For all small interfering RNAs, there was a decrease in the phosphorylation of Akt after LPS treatment that correlated with the depletion level of PI3K $\gamma$ (Fig. 5a). As a second approach, macrophages were treated with a PI3K $\gamma$ isoform-specific inhibitor, AS605240 (ref. 35), resulting in reduced phosphorylation of Akt in a dose-dependent manner (Fig. 5b). As PI3K $\gamma$ inhibitors can have off-target effects at higher concentrations, we also used genetic ablation of PI3K $\gamma$ in knockout mice to assess its function. Targeted knockout of the p110 $\gamma$ subunit $\left(\mathrm{PI} 3 \mathrm{~K} \gamma^{-/}\right)$in the C57BL/6 mouse background does not affect the expression of other p110 isoforms $(\alpha, \beta \text { and } \delta)^{28}$. Primary bone marrowderived macrophages (BMMs) from PI3K $\gamma^{-1}$ and wild-type mice were challenged with LPS, resulting in $>50 \%$ decreased phosphorylation of Akt in PI3K $\gamma$-null cells (Fig. 5c). Taken together, these findings indicate that loss of PI3K $\gamma$ in LPStreated macrophages significantly reduces Akt signalling. This effect mimics the knockdown of Rab8a (see Fig. 2a,b) and is consistent with the notion that PI3K $\gamma$ acts as the Rab8a effector during TLR4 signalling. We therefore deduce that the 
a

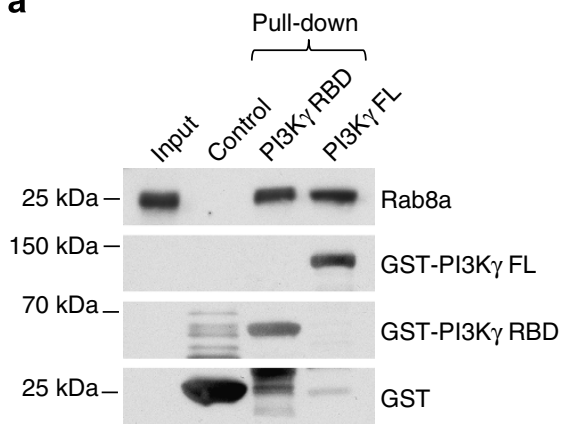

C
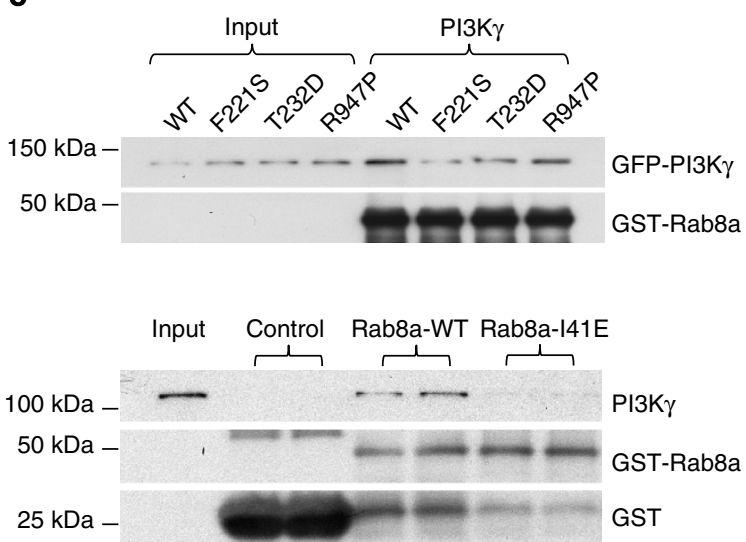

b

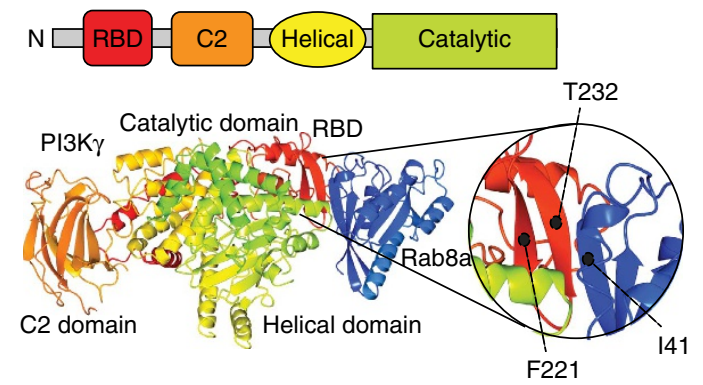

d
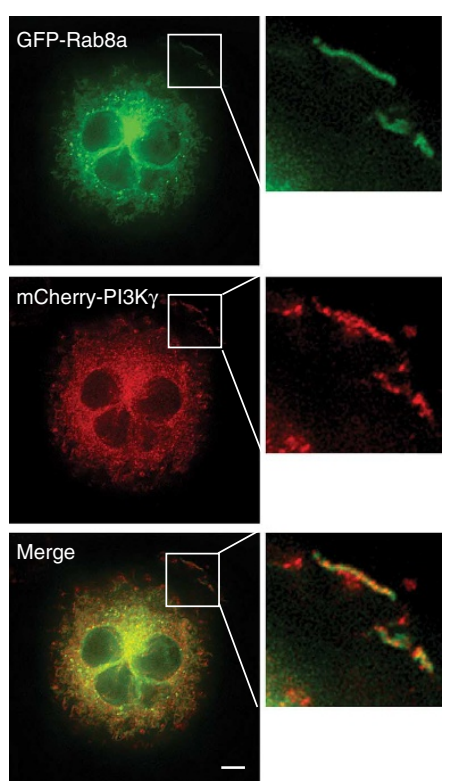

Figure 4 | Characterization of Rab8a and PI3K $\gamma$ interaction. (a) Bacterially expressed GST-PI3K $\gamma$ RBD (amino acids 1-356) and insect cell-expressed fulllength GST-PI3K $\gamma$ were used to pull down Rab8a from RAW 264.7 cell lysates. (b) PI3K $\gamma$ domain structure and homology model of the Rab8a/PI3K $\gamma$ complex. The homology model was generated using the crystal structure of GTP-bound Rab8a (PDB:3QBT) and the co-crystal of PI3K $\gamma$ and Ras (PDB:1HE8). (c) Pairwise GST pull-down experiment using either the mutant forms of PI3K $\gamma$ with wild-type Rab8a or mutated Rab8a with wild-type PI3K $\gamma$. All Rab8 fusion proteins were GTP-loaded. The kinase-inactive mutant (R947P) was used as a negative control. (d) LPS-treated (15 min) RAW 264.7 macrophages cotransfected with GFP-Rab8a and mCherry-PI3K $\gamma$ show colocalization in ruffles. Scale bar, $10 \mu \mathrm{m}$. All immunoblots were cropped for presentation; full-length blots are provided in Supplementary Fig. 5.

physiological roles of both Rab8a and PI3K $\gamma$ are to augment TLR4-mediated PI3K/Akt signalling.

We also examined the downstream cytokine responses to LPS in $\mathrm{PI} 3 \mathrm{~K} \gamma^{-/-}$BMMs, where production of pro-inflammatory cytokines, IL-6 and IL-12p40, is significantly enhanced in response to LPS, compared with BMMs from control animals (Fig. 6a,b). In contrast, the synthesis and release of IL-10 and the anti-viral type I interferon, IFN- $\beta$, are significantly suppressed in PI3K $\gamma^{-/-}$BMMs. Hence, PI3K $\gamma$ affects not only signalling, but also the innate immune responses activated by TLR4 in macrophages. In keeping with the signalling profiles and with the role of Rab8a, PI3K $\gamma$ serves to bias the inflammatory response by constraining the production of pro-inflammatory cytokines.

PI3K $\gamma$ does not regulate endocytosis of TLR4. The internalization of TLR4 (ref. 12) by effectively removing it from the surface adaptors, Mal and MyD88, is one possible mechanism for limiting pro-inflammatory signalling ${ }^{10}$. Since class I PI3Ks are commonly involved in endocytic pathways ${ }^{36}$ and Rabs are also common endocytic regulators ${ }^{37}$, a plausible mechanism for the actions of $\mathrm{Rab} 8 \mathrm{a} / \mathrm{PI} 3 \mathrm{~K} \gamma$ would ostensibly be to regulate the endocytosis of TLR4. However, testing of known endocytic pathways in $\mathrm{PI} 3 \mathrm{~K} \gamma^{-/}$BMMs revealed no requirement for $\mathrm{PI} 3 \mathrm{~K} \gamma$ in directly regulating $\mathrm{Fc} \gamma \mathrm{R}$-mediated phagocytosis, clathrin-mediated transferrin receptor endocytosis or dextranlabelled macropinocytosis (Supplementary Fig. 4a-c). Thus, common endocytic pathways in PI3K $\gamma$-null mice appear to operate efficiently. The internalization of TLR4 itself was then measured by surface immunolabelling and flow cytometry over a time course of LPS activation. TLR4 is successfully internalized in PI3K $\gamma^{-/}$BMMs at the same rate as in control cells (Fig 7a,b). Hence, PI3K $\gamma$ is not required to regulate the endocytosis of TLR4 in this context.

PI3K $\gamma$ mediates cytokine biasing through mTOR. A second mechanistic explanation for the cytokine bias observed in Rab8a- and PI3K $\gamma$-depleted cells involves the mTORC1 complex, which acts downstream of PI3K in TLR4 pathways. Within this 
a
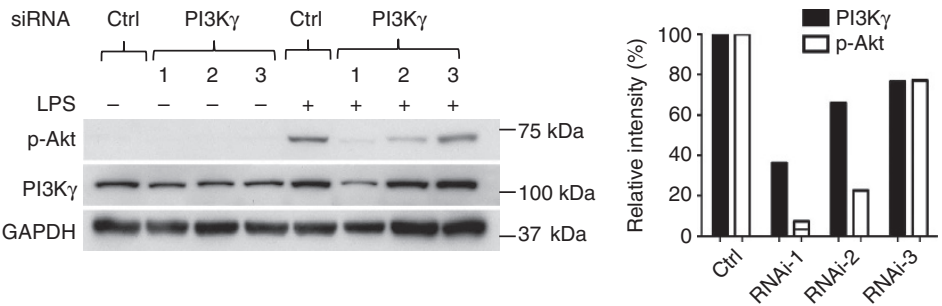

b
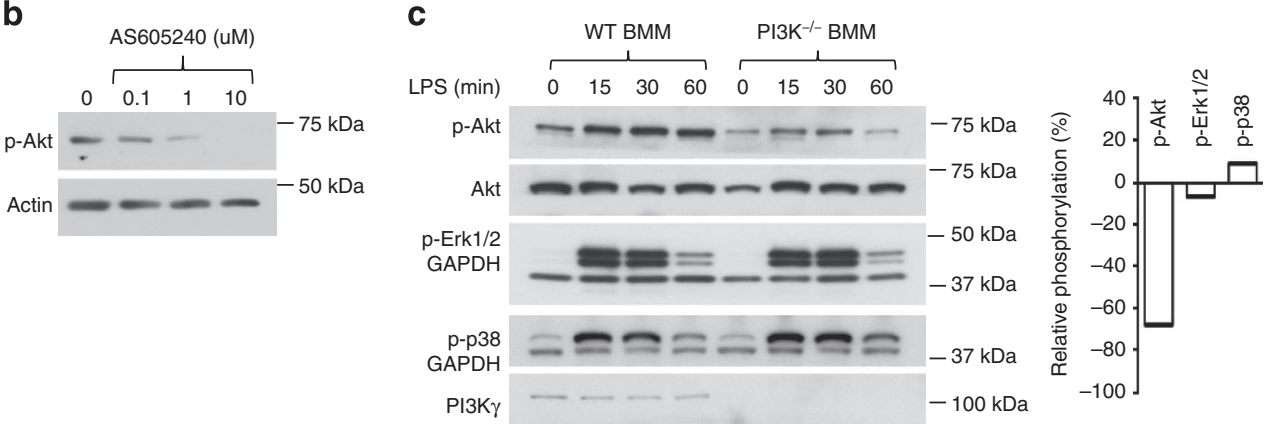

Figure 5 | PI3K $\gamma$ regulates TLR4-mediated signalling in macrophages. (a) RAW 264.7 macrophages treated with either control (non-targeting), or one of three independent PI3K $\gamma$-targeting small interfering RNAs were incubated with LPS for 30 min. Quantification of western blots was performed using densitometry. (b) RAW 264.7 cells were treated for 30 min with either vehicle (DMSO), 0.1, 1 or $10 \mu \mathrm{M}$ of AS605240 before addition of LPS for 30 min. Cell extracts were analysed for Akt phosphorylation. (c) BMMs derived from wild-type (WT) and PI3K $\gamma^{-/-}$animals were subjected to a 60-min time course of LPS. Cell extracts were analysed for Akt, ERK1/2 and p38 phosphorylation. Quantification of phosphorylation is at the 30-min time point only and the gel is representative ( $n=3$ each). All immunoblots were cropped for presentation; full-length blots are provided in Supplementary Fig. 6 .
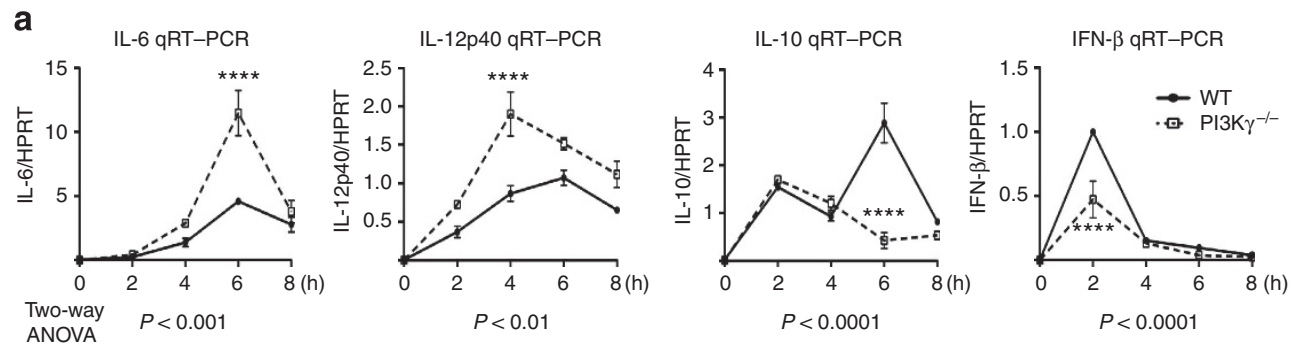

b

IL-6 secretion

IL-12p40 secretion

IL-10 secretion

IFN- $\beta$ secretion (8 h)
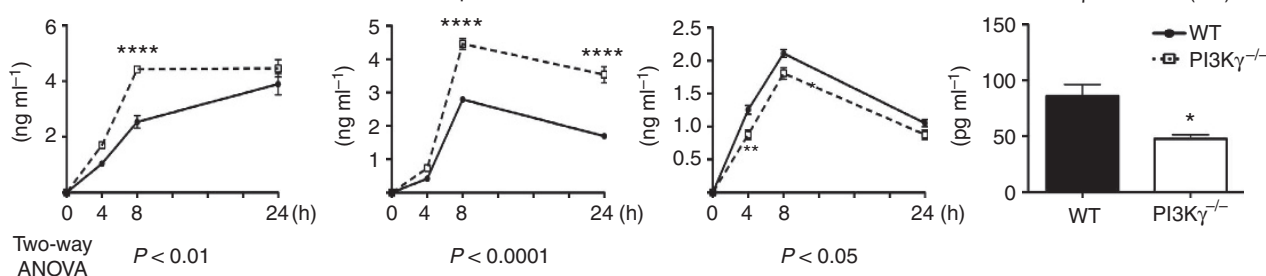

Figure 6 | PI3K $\gamma$ attenuates the inflammatory response. (a) Quantitative reverse transcriptase PCR time course after LPS treatment in WT and $\mathrm{PI} 3 \mathrm{~K}^{-}{ }^{-/}-\mathrm{BMMs}$. Data points are the mean \pm s.e.m. $(n=3)$ of cytokine profiles and were compared by two-way analysis of variance (ANOVA). (b) Cytokines secreted into the BMM medium were measured by enzyme-linked immunosorbent assay, post-LPS treatment, with the exception of IFN- $\beta$, which was measured at $24 \mathrm{~h}$. Each data point is the mean \pm s.e.m. $(n=3$ with three replicates). Significance was measured by two-way ANOVA with Sidak's post-test for multiple comparisons ( ${ }^{\star \star} P<0.01$, $\left.{ }^{\star \star \star \star} P<0.0001\right)$. IFN- $\beta$ was measured by Student's $t$-test.

complex, the serine/threonine protein kinase mTOR has been implicated in the transcriptional and post-translational regulation of cytokine production and biasing ${ }^{38}$. In our wild-type BMMs, inhibition of mTORC1 with rapamycin effectively enhances the production of pro-inflammatory cytokines, IL- 6 and IL-12p40, while decreasing the levels of IL-10 (Fig. 8a). This is roundly consistent with the effects of Rab8a and PI $3 \mathrm{~K} \gamma$ on cytokine outputs in our experiments, and with findings relating to mTOR function in the literature ${ }^{16,39}$. To investigate if PI3K $\gamma$ regulates
mTOR downstream of TLR4, we analysed the phosphorylation of Akt and mTOR in LPS-treated wild-type and PI3K $\gamma^{-1}$ BMMs (Fig. 8b). Quantification revealed a significant reduction in both Akt and mTOR phosphorylation in the absence of $\mathrm{PI} 3 \mathrm{~K} \gamma$ (Fig. 8b). To investigate the significance of the decreased mTOR phosphorylation, we tested two canonical mTORC1 substrates, 4E-BP1 and p70S6K, known targets during LPS stimulation $^{16}$. In PI $3 \mathrm{~K}^{-}{ }^{-}-$cells after LPS treatment, the phosphorylation of p70S6K was reduced to below detectable 
a

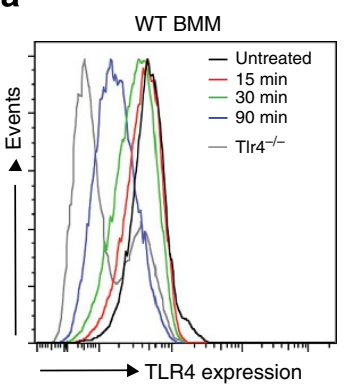

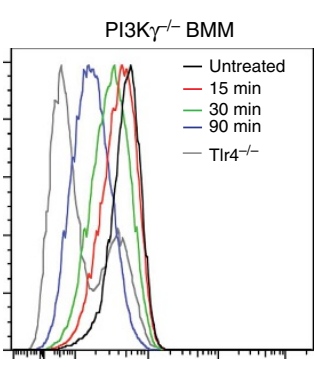

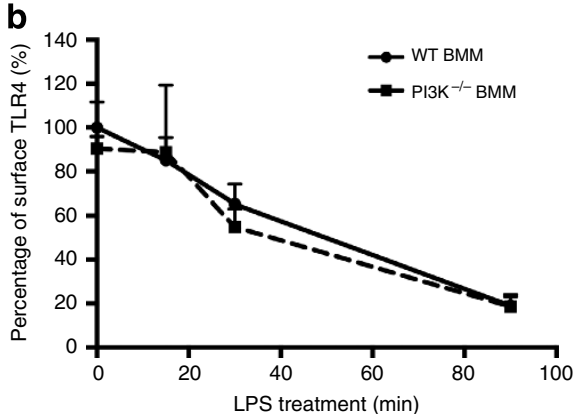

Figure 7 | Endocytosis of surface TLR4 is not PI3K $\gamma$-dependent. (a) Untreated or LPS-treated (100 ng ml ${ }^{-1}$ for the times indicated) WT and PI3K $\gamma^{-/-}$ BMMs were used to examine surface levels of endogenous TLR4 by immunostaining and flow cytometry. (b) Quantification of TLR4 internalization over time. The percentage of surface TLR4 was calculated by the mean fluorescent intensity of TLR4 receptor staining at each time point over MFI of untreated cells ( $n=3$ with three replicates).

a
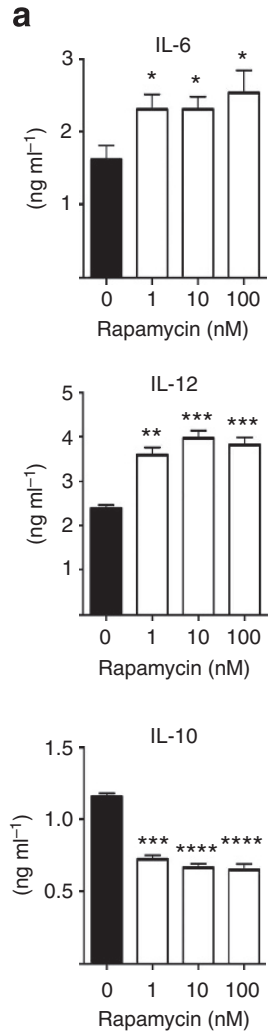

b

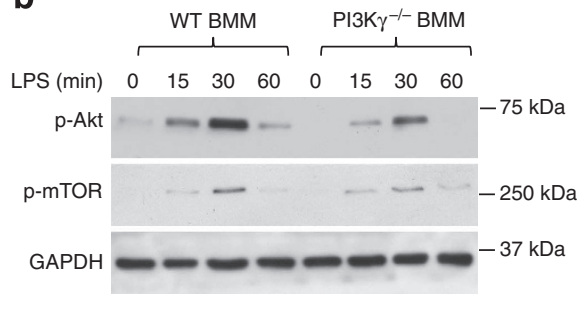

C

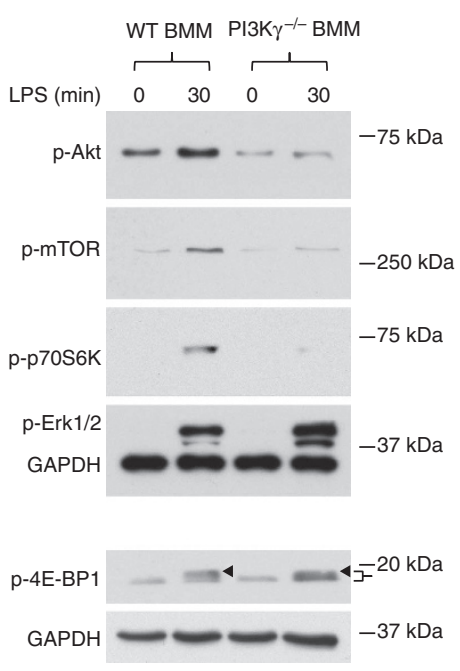

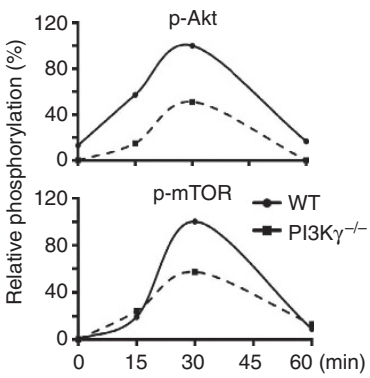

Figure 8 | PI3K $\gamma$ regulation of mTOR during TLR4 signalling. (a) Cytokines secreted into the medium of untreated and mTOR inhibitor (rapamycin)treated BMMs were measured by enzyme-linked immunosorbent assay, post-LPS treatment at $8 \mathrm{~h}$. Data points are the mean \pm s.e.m. $(n=5$ with three replicates). Significance was measured Student's $t$-test $\left({ }^{\star} P<0.05,{ }^{\star \star} P<0.01,{ }^{\star \star \star} P<0.001,{ }^{\star \star \star \star} P<0.0001\right)(\mathbf{b})$ BMMs derived from WT and PI3K $\gamma{ }^{-/}-$ animals were subjected to a 60-min time course of LPS. Cell extracts were analysed for Akt and mTOR phosphorylation. Densitometric quantification of phosphorylation is at the 30-min time point only and the gel is representative ( $n=3$ each). (c) BMMs derived from WT and PI3K $\gamma^{-/-}$animals were treated with LPS (30 min) and cell extracts were analysed for phosphorylation of the mTOR substrates, p70S6K and 4E-BP1. Arrow heads indicate the hyperphosphorylated form of $4 \mathrm{E}-\mathrm{BP1}$, which has a mobility shift. All immunoblots were cropped for presentation; full-length blots are provided in Supplementary Fig. 6.

levels, while 4E-BP1 phosphorylation was reduced for the higher molecular weight isoform. Together, these results confirm a defect in the PI3K/Akt/mTOR pathway (Fig. 8c). Thus, PI3K $\gamma$ is required for efficient activation of mTOR in response to pathogen recognition by TLR4. Taken together, our data suggest a new role for PI3K $\gamma$ during TLR4 signalling and one that is independent of receptor endocytosis. The recruitment of Rab8a and $\mathrm{PI} 3 \mathrm{~K} \gamma$ during TLR4 activation functions to enhance the mTOR signalling pathway, effectively polarizing the cytokine response and limiting inflammation.

\section{Discussion}

In this study, we describe a new regulatory unit consisting of Rab8a with PI3K $\gamma$ as its effector. In this context, Rab8a plays a new role in signalling with novel PI $3 \mathrm{~K} \gamma$ function in the TLR4 pathway. This additional role for PI $3 \mathrm{~K} \gamma$ extends its reach beyond known GPCRs and RTKs. In macrophages exposed to LPS, Rab8a and $\mathrm{PI} 3 \mathrm{~K} \gamma$ are recruited to dorsal ruffles along with components of the TLR4/Mal/MyD88 complex. Rab8a and PI3K $\gamma$ are two newly introduced partners that are required for effective Akt/mTOR signalling during TLR4 activation. PI3K $\gamma$ serves to 
enhance mTOR phosphorylation and signalling via this arm of the TLR4 response, whereby downstream proteins can both constrain inflammatory responses by curtailing production of pro-inflammatory cytokines and bias the response in favour of regulatory or anti-inflammatory cytokines ${ }^{38}$. Our findings thus reveal the Rab8a/PI3K $\gamma$ complex as an important and additional mechanism for controlling PI3K signalling in inflammation.

Rab8a is a ubiquitous Rab, functioning in multiple recycling pathways through a variety of effectors ${ }^{27,40,41}$. The Rab8a localization we show in activated macrophages is broadly commensurate with its previous localization on cell surfaces, at leading edges and on membrane vesicles and tubules moving to and from the surface ${ }^{23,40}$. Within this distribution, we show a specific and dramatic recruitment of Rab8a to dorsal ruffles on the surface of LPS-activated macrophages, along with components of the surface TLR4 complex. Our study serves to highlight macrophage dorsal ruffles as a previously unheralded site for clustering of the TLR4 receptor and associated molecules. The features we show, namely the recruitment of PI $3 \mathrm{~K} \gamma$, generation of the signalling phosphoinositide $\mathrm{PI}(3,4,5) \mathrm{P} 3$ and subsequent activation of Akt, denote these dorsal ruffles as a functional site for TLR4 signalling. Rab8a enrichment in the ruffles is striking and reflects the clustering of Rabs at functional membrane domains, mostly at the behest of their guanine nucleotide exchange factors and effectors. The identity of such Rab8a recruitment factors in this locale is not yet known, although the GTPase Arf6 is a candidate player due to its roles in ruffle formation ${ }^{42}$, its deactivation of Rab8a in epithelial cells ${ }^{43}$ and a proposed role in TLR4 activation in macrophages ${ }^{13}$. While Rab8a regulates signalling from the surface TLR4 complex, additional Rabs influence TLR4 at other points along the endocytic and recycling pathways via more traditional trafficking mechanisms. Rab1la regulates recycling endosomemediated delivery of TLR4 to phagosomes, an alternative site proposed for LPS activation of TLR4 wherein TLR4 signals via the TRIF/TRAM adaptors to produce IFN- $\beta^{5}$. Of note is the fact that phagosomes can be generated from dorsal ruffles, suggesting perhaps another route for spatiotemporal regulation of TLR4. Also, Rab10 (ref. 3) and Rab7 (ref. 4) function in recycling of TLR4 to the surface and for degradation, respectively; however, Rab effectors relevant to TLR4 at all of these other steps remain undefined. We now recast Rab8a with a new effector and a very different role through its direct involvement in PI3K signalling in the TLR4 pathway.

$\mathrm{PI} 3 \mathrm{~K} \gamma$ was revealed herein as an unexpected binding partner for Rab8a and the first instance of class 1B PI3Ks directly interacting with a Rab. Rab5 was previously implicated as an effector for class IA PI3K $\beta^{44}$, although the nature of this interaction was not characterized. At a molecular level, the PI3K catalytic subunit p110 $\gamma$ is traditionally recruited by binding directly to Ras, along with a class IB regulatory subunit, and the G $\beta \gamma$ subunit of an activated GPCR complex ${ }^{45}$. We now confirm that Rab8a and PI3K $\gamma$ bind directly to each other, using homology modelling and mutagenesis of critical residues. Rab8a binds to two residues in the RBD of PI3K $\gamma$ that are critical and necessary for Ras binding to PI3K $\gamma^{34}$. Recently, Rho family GTPases, Racl and CDC42, but not Ras, were also shown to directly interact with class IA PI $3 K \beta$ through the kinase's $R_{B D}{ }^{46}$. Our findings now show that the PI3K RBD also supports interactions with a Rab GTPase, in addition to Ras and Rho family GTPases ${ }^{46}$. These $\mathrm{G}$ protein families thus offer a variety of modalities for the recruitment and regulation of PI3Ks in signalling pathways.

In the context of LPS signalling, it remains unclear precisely how PI3K $\gamma$ is recruited and activated by TLR4, particularly because PI $3 \mathrm{~K} \gamma$ does not bind directly to TLR4. Our findings suggest that Rab8a provides a parallel means of recruiting a PI3K to augment LPS signalling, with Rab8a/PI3K $\gamma$ acting as an auxiliary unit in its own right or in the context of another receptor. Such crosstalk for amplifying PI3K-mediated signalling has precedents, including the FceRI receptor in mast cells, where this receptor has no direct link to GPCRs, yet degranulation is dependent on $\mathrm{PI} 3 \mathrm{~K} \gamma^{47}$. Indeed, a seminal study of tumourassociated macrophages expanded the field of PI3K $\gamma$ 's influence by showing that it regulates signalling not only from GPCRs, but also from RTKs and the TLR/IL1 receptor ${ }^{29}$. In the complex ligand environment of tumour inflammation, the loss of $\mathrm{PI} 3 \mathrm{~K} \gamma$ was found to limit tumour progression ${ }^{29}$, in line with the current interest in PI $3 \mathrm{~K} \gamma$ inhibitors for treating cancer. By adding the pathogen recognition receptor, TLR4, to the classes of receptors employing PI $3 \mathrm{~K} \gamma$, we raise questions for the future about even more receptors that may activate $\operatorname{Rab} 8 \mathrm{a} / \mathrm{PI} 3 \mathrm{~K} \gamma$, including other members of the TLR family and their arrays of pathogen-derived ligands. Further studies are also needed to establish whether Rab8a, rather than Ras, is involved in recruiting PI3K $\gamma$ to other receptor families.

Previous studies have focused on TLR4 internalization as a mechanism by which macrophages can terminate the production of pro-inflammatory cytokines ${ }^{10-12}$. The class IA $\mathrm{PI} 3 \mathrm{~K} \delta$ is recruited to the TLR4 complex, where it reportedly regulates internalization of the receptor by an unknown pathway ${ }^{18}$. This results in a switch from cell surface MyD88/Mal signalling, which generates pro-inflammatory cytokines, to the endosomal adaptors TRIF/TRAM, which induce production of type I interferons ${ }^{10}$. We saw no general effects on endocytosis or the internalization of TLR4 itself after loss of PI3K $\gamma$. Therefore, Rab8a/PI3K $\gamma$ operates via another mechanism to control TLR4 signalling and cytokine production. The effects we demonstrate here for $\mathrm{PI} 3 \mathrm{~K} \gamma$ are similar to those reported for PI3K $\delta$ in enhancing Akt signalling and in limiting pro-inflammatory cytokines. Therefore, it is likely that both PI3Ks act in concert to augment the same effect, possibly reflecting the need for multiple mechanisms to stimulate and fine-tune the production of inflammatory cytokines.

Mechanistically, we found that Rab8a and PI3K $\gamma$ exert their control over cytokine production by signalling through the multifunctional serine/threonine protein kinase mTOR, which acts as a hub downstream of TLR4 to strategically bias cytokine responses ${ }^{48}$. In this context, mTOR signalling inhibits the function of $\mathrm{NFKB}$ and the transcription of pro-inflammatory cytokines, IL- 6 and IL-12p40, while enhancing the function of STAT3 and the transcription of an anti-inflammatory or regulatory cytokine, IL-10 (refs 16,49). The importance of mTOR in this regulatory role emerged with a recent study showing that the intracellular pathogen Legionella pneumophila subverts the immune response with bacterial effectors that target mTOR to disengage its cytokine biasing role ${ }^{39}$. The regulation of mTOR downstream of TLR4 by PI3K $\gamma$ results in the polarized inflammatory response towards an anti-inflammatory setting. This effect has been demonstrated elsewhere, through the pharmacological inhibition of mTORC1 with rapamycin and by the genetic perturbation of TSC2-another kinase in the pathway ${ }^{16}$. Our findings represent the first example of mTOR regulation by a Rab GTPase through PI $3 \mathrm{~K} \gamma$. Given that mTOR controls many cellular functions, Rab8a and PI3K $\gamma$ may have broader physiological effects beyond cytokine production.

$\mathrm{PI} 3 \mathrm{~K} \gamma$ has become an attractive drug target in cancer and other diseases ${ }^{50}$. PI $3 \mathrm{~K}^{-1}{ }^{-}$mice show reduced inflammation in a number of disease models, including diabetes ${ }^{51}$ and cardiovascular disease ${ }^{52}$, primarily related to roles for this kinase in GPCR-dependent cell migration of immune cells and activation of the respiratory burst ${ }^{28}$. On this basis, PI3K $\gamma$ inhibitors are also viewed as potential anti-inflammatory drugs ${ }^{4}$. However, at least 
in the context of bacterial infection, our preliminary results suggest that $\mathrm{PI} 3 \mathrm{~K} \gamma$ inhibition might acutely skew signalling towards pro-inflammatory cytokines, enhancing bactericidal responses, but exacerbating inflammation. Further studies are now needed to dissect the role of $\mathrm{PI} 3 \mathrm{~K} \gamma$ and the clinical consequences of its inhibition over the course of bacterial infection and in disease. As a pharmacologically tractable target operating at a critical point to regulate cytokine outputs from TLR4 activation, understanding how PI3K $\gamma$ influences infection and inflammatory disease is vital.

\section{Methods}

Antibodies and reagents. Primary antibodies recognizing PI $3 \mathrm{~K} \alpha, \beta, \gamma, \delta$ (sampler kit-9655), phospho-mTOR, phospho-4E-BP, phospho-p70 S6 kinase (sampler kit-9862), Akt (9272), phospho-Akt $\left(\mathrm{Ser}^{473}\right)$ (4962), phospho-p38 MAPK (Thr ${ }^{180 /}$ $\mathrm{Tyr}^{182}$ ) (4511), IкB $\alpha$ (9242), phospho-ERK1/2 (4370) were purchased from Cell Signaling Technology (Beverly, MA, USA). Mouse anti-Rab8 (610845) was purchased from BD Transduction Laboratories (Lexington, KY, USA). GST antibody (71-7500) was purchased from Invitrogen, Sydney, Australia. Anti-TLR4 for western blotting was from Abcam (ab22048). For fluorescent-activated cell sorting (FACS) analysis, the anti-TLR4 SA15-21 clone was biotinylated using the EZ-Link Micro Sulfo-NHS-LC-Biotinylation Kit (Thermo Scientific, CA, USA). Streptavidin-APC (Biolegend, CA, USA) was used to detect biotinylated antiTLR4. Mouse anti-glyceraldehyde-3-phosphate dehydrogenase (2275-PC-1) was purchased from Trevigen (Gaithersburg, MD, USA). Alexa Fluor 488/594(A21208) and 647- (A31573) conjugated secondary antibodies, Alexa Fluor 488- and 647-dextran and wheat germ agglutinin (WGA)-Texas Red were purchased from Molecular Probes (Invitrogen, OR, USA). Horseradish peroxidaseconjugated goat anti-mouse and rabbit antibodies (81-6520) were obtained from Zymed (San Francisco, CA, USA). Bacterial lipopolysaccharide (LPS), purified from Salmonella enterica serotype Minnesota Re 595, was purchased from SigmaAldrich (Castle Hill, NSW, Australia). AS605240 $\left(\mathrm{IC}_{50}=0.008 \mu \mathrm{M}\right)$ was purchased from Sigma-Aldrich and IC87114 $\left(\mathrm{IC}_{50}=0.07 \mu \mathrm{M}\right)$ was from Scientifix, Australia. For phagocytosis, human IgG (Invitrogen) was conjugated to $3 \mu \mathrm{m}$ latex beads (Sigma). All other chemicals and reagents were from Sigma-Aldrich.

Plasmid and constructs. The mouse PI $3 \mathrm{~K} \gamma \mathrm{cDNA}$ was obtained from the Facility for Life Science Automation at the Institute for Molecular Bioscience. GST-PI3K $\gamma$ FL and His-PI3K $\gamma$-FL were expressed in Sf9 insect cells using baculovirus expression vector systems according to the manufacturer's instructions (Invitrogen). Rab8a was subcloned into pEGFP-C1, pm-Cherry-C1 and ptd-Tomato-C1 that had been generated from pCMV-tdTomato (Clontech). The pEGFP-Rab8aCAAX domain (Rab8a tail) was generated by PCR to contain the last 29 amino acids of the Rab8a C terminus with a modification of the CAAX motif to CMIV to allow for prenylation of the fusion protein ${ }^{53,54}$. Rab8a was also subcloned into pGEX6P-1 and expressed in $E$. coli ${ }^{55}$. Phosphoinositide probes and pEGFP-C1-Akt-PH were kindly provided by Frederic Meunier, and the lentiviral vector PLL5.0 was obtained from Alpha Yap (both of The University of Queensland, QLD, Australia). Mal-Cerulean and TLR4-Citrine plasmids were gifts from Nicholas J. Gay, University of Cambridge, UK.

Expression and purification of recombinant proteins. Recombinant GST-tagged proteins were produced in an E. coli BL21(DE3) strain transformed with the plasmid DNA of interest. Expression of the recombinant protein was induced with the addition of $0.5 \mathrm{mM}$ IPTG for $18 \mathrm{~h}$ at $25^{\circ} \mathrm{C}$ with shaking. The bacterial cells were then pelleted by centrifugation at $5,000 \mathrm{~g}$. The pellets were resuspended in icecold lysis buffer (Tris pH 7.4, $300 \mathrm{mM} \mathrm{NaCl}, 1 \mathrm{mM}$ EDTA, cOmplete EDTA-free protease inhibitor cocktail tablet (Roche Applied Science), $1 \mathrm{mM}$ DTT and $1 \mathrm{mM}$ PMSF). The bacterial lysates were lysed using a Constant Systems cell disruptor according to the manufacturer's instructions (Thermo Scientific, Australia) and then cleared by centrifugation at $48,254 \mathrm{~g}$ at $4{ }^{\circ} \mathrm{C}$. The recombinant proteins were affinity purified by incubating the cleared lysate with Glutathione Sepharose (GE Healthcare Life Science, Australia) for $1 \mathrm{~h}$ at $4^{\circ} \mathrm{C}$. The beads were washed extensively with $1 \mathrm{M} \mathrm{NaCl}$ in $20 \mathrm{mM}$ Tris $\mathrm{pH}$ 7.4. GST-fusion proteins on the beads were stored at $-20^{\circ} \mathrm{C}$ in $20 \mathrm{mM}$ Tris $\mathrm{pH} 7.4$ containing $20 \%$ glycerol.

\footnotetext{
Mouse model. Knockout of the p110 $\gamma$ subunit in PI3K $\gamma^{-/-}$mice (pik3cg2/2) on a C57BL/6 background has been previously described ${ }^{28}$. Age (16-20 weeks) and sexmatched C57BL/6 mice were used as controls. Mice were housed and used for experiments in accordance with approved animal ethics protocols at AMREP (Alfred Medical Research and Education Precinct) AEC, Alfred Hospital Melbourne, Victoria. (E/1089/2011/M). Experiments utilized samples or cells from three or more mice per group per time point to generate statistical significance. Animals within groups were randomized for treatments. Primary BMMs were obtained by ex vivo differentiation of bone marrow cells collected from mouse femurs or tibias. Cells were differentiated for 7 days in complete RPMI medium supplemented with
}

$20 \mathrm{U} \mathrm{ml}^{-1}$ penicillin, $20 \mu \mathrm{g} \mathrm{ml}^{-1}$ streptomycin and $100 \mathrm{ng} \mathrm{ml}^{-1}$ purified recombinant macrophage colony-stimulating factor-1 (refs 56,57).

Cell culture and siRNA. The mouse RAW 264.7 macrophage cell line was sourced from ATCC. PI3K $\gamma$ was silenced using Stealth siRNA primer sets from Life Technologies, Australia (catalogue number 1320003). RAW 264.7 macrophages were cultured in RPMI 1640 medium (Lonza) supplemented with $10 \%$ heatinactivated FCS (Thermo Trace) and $2 \mathrm{mM}$ L-glutamine (Invitrogen) in humidified $5 \% \mathrm{CO}_{2}$ at $37^{\circ} \mathrm{C}$ (ref. 58). Cells were screened monthly for mycoplasmal contamination. For transient expression of cDNA, cells at $50 \%$ confluence were transfected using Lipofectamine 2000 (Invitrogen) according to the manufacturer's instructions. Cells were typically used for experiments $18 \mathrm{~h}$ after transfection. Nontargeting siRNA was used as a control. For Rab8a depletion, an 18 nucleotide shRNA was designed and cloned into the lentivirus expression vector LentiLox pLL5.0 (refs 59-61), using forward primer $5^{\prime}$-tGCCTTCAACTCCAC ATT CAttcaagagaTGAATGTGGAGTTGAAGGCttttttg- $3^{\prime}$, and reverse primer $5^{\prime}$-TCG ACAAAAAAGCCTTCAACTCCACATTCATCTCTTGAATGAATGTGGATTG AAGGCA-3'. The shRNA was cloned downstream of the U6 promoter (HpaI and XhoI) into a lentivirus expression vector pLL5.0 (backbone pLL3.7) carrying a soluble mCherry gene as a fluorescent reporter. The pLL5.0 shRNA and packaging vectors were transfected into HEK-293T cells by Lipofectamine 2000 precipitation. Virus-like particles were collected from the supernatant $48-72 \mathrm{~h}$ after transfection, and virus was concentrated using polyethylene glycol 6000 (ref. 62). Aliquots of virus were subsequently used for titration or stored at $-80^{\circ} \mathrm{C}$. Titres were determined by infecting HEK-293T cells with serial dilutions of concentrated lentivirus. RAW 264.7 macrophages were infected with lentiviral particles at a multiplicity of infection of 10 per cell. Cells were incubated at $37^{\circ} \mathrm{C}$ with lentivirus in RPMI complete medium and collected $48 \mathrm{~h}$ post infection. Single-cell suspensions were sorted by flow cytometry according to moderate to high levels of reporter gene expression.

Fixed cell ruffle quantification and analysis. RAW 264.7 macrophages were grown on coverslips overnight at a low confluency. Cells were either treated with exogenous LPS $100 \mathrm{ng} \mathrm{ml}^{-1}$ (stimulated) or not treated (unstimulated) for $30 \mathrm{~min}$ before fixation. Cytoskeletal F-actin was labelled with Alexa Fluor 488 phalloidin (Life Technologies) for $60 \mathrm{~min}$; in addition, nuclei were labelled with 4',6-diamidino-2-phenylindole (DAPI) concurrently. Three-dimensional $z$-stacks were acquired using a DeltaVision devolution microscope (GE Healthcare) and were analysed using Fiji image software. The mid-point of cellular nuclei (DAPI) was selected and labelled as the new base slice. Slices from the mid-point until the top of the cell were flattened using maximum projection. Background subtraction and median filters were applied to the flattened image for smoothing purposes for further analysis. The image was then duplicated, whereby a threshold mask was constructed (equally for all images) and all regions collected from the phalloidin threshold image were selected as the region of interest (ROI). The fluorescence intensity for the generated ROI in the phalloidin channel was then measured and quantified using GraphPad Prism.

Phagocytic assay and procedures. Wild-type and PI3K $\gamma^{-/}$BMMs $(n=3$ each) were grown on coverslips before phagocytosis experiments. Latex beads ( $3 \mu \mathrm{m}$ diameter) from Sigma-Aldrich (LB30-2ML) were diluted $1 \times 10^{-1}$ in $\mathrm{dH} 2 \mathrm{O}$ and washed with phosphate-buffered saline (PBS) three times by centrifugation. Beads were resuspended in $2 \mu \mathrm{g} \mathrm{ml}^{-1}$ human immunoglobulin $\mathrm{G}$ (IgG) (Invitrogen, 02-7102) in PBS. IgG was left to bind by passive adsorption for $2 \mathrm{~h}$ at room temperature on a rotating mixer. The suspension was then washed three times and resuspended in PBS and sodium azide $(0.2 \%)$. For phagocytosis, $1 \mu \mathrm{l}$ of this suspension was added to macrophages on coverslips in $1 \mathrm{ml}$ of cold complete RPMI medium, and spun onto the cells at $1,00 \mathrm{~g}$ for $2 \mathrm{~min}$ at $4{ }^{\circ} \mathrm{C}$. Cells are then transferred back to $37^{\circ} \mathrm{C}$ for $5-20 \mathrm{~min}$. Cells were fixed in $4 \%$ paraformaldehyde for $30 \mathrm{~min}$ and then permeabilized, if required, with $0.1 \%$ Triton X-100/PBS for $5 \mathrm{~min}$. Washing, blocking and antibody incubation steps were done using blocking buffer (0.5\% BSA/PBS). Human IgG on beads was detected using Cy3 goat antihuman antibody (1:200, Jackson Labs 109-165-003). Cells were labelled with Alexa350/488 phalloidin (1:50/1:500, Molecular Probes, 22281,12379), to outline cells and show actin-enrichment at sites of phagocytosis. Coverslips were mounted in Prolong Gold (Invitrogen, P10144) for imaging. Olympus BX-51 upright microscope fitted with an Olympus DP-71 12Mp Colour Camera. Images were captured using a $\times 40 / 1.35$ U Apo Oil lens with the Olympus DP controller version 2.1 capture software at an image size of $2040 \times 1536$ pixels. Each bead was classified as either phagocytosed $(\mathrm{Cy} 3<5 \%)$, in-between $(5 \%<\mathrm{Cy} 3<95 \%)$ or attached $(\mathrm{Cy} 3>95 \%)$ for comparison between groups ${ }^{63}$. $(n>200$ beads per animal).

TLR4 internalization assay. Wild-type and PI3K $\gamma^{-/-}$BMMs $(n=3$ each) were grown on non-adherent tissue culture plates. In FACS tubes, $0.5 \times 10^{6} \mathrm{cells} \mathrm{ml}^{-1}$ for each animal in triplicate were treated with or without LPS $\left(100 \mathrm{ng} \mathrm{ml}^{-1}\right)$ for indicated times at $37^{\circ} \mathrm{C}$ before being placed on ice to halt all internalization. Cells were washed twice with ice-cold FACS buffer (PBS, 1\% FCS, 5 mM EDTA) and blocked with $24 \mathrm{G} 2$ for $20 \mathrm{~min}$ on ice. Cell surface TLR4 was stained with 
biotinylated anti-TLR4 (clone SA15-21 Biolegend 145402) at $0.5 \mu \mathrm{g} \mathrm{ml}^{-1}$. Cells were washed three times in FACS buffer before labelling with Streptavidin-APC (Biolegend 405207) at $0.5 \mu \mathrm{g} \mathrm{ml}^{-1}$. Cells were washed extensively in FACS buffer at $4{ }^{\circ} \mathrm{C}$ before acquisition on a Canto II cytometer (Becton Dickinson). Cellular profiles were analysed using FlowJo software (TreeStar). The percentage internalization $=\left(\mathrm{MFI}^{\mathrm{X}}-\mathrm{MFI}^{\mathrm{TLR} 4-I-}\right) /\left(\mathrm{MFI}^{\mathrm{WT}}\right.$ untreated $\left.-\mathrm{MFI}^{\mathrm{TLR} 4-I-}\right) \times 100$ for each data point.

Transferrin uptake assay. Wild-type and PI $3 \mathrm{~K}^{-/-}$BMMs ( $n=3$ each) grown on coverslips were serum starved for $30 \mathrm{~min}$ and treated with Alexa Fluor 546labelled mouse transferrin (Life Technologies) for $10 \mathrm{~min}$ at $37^{\circ} \mathrm{C}$. Cells were then washed and chased for $20 \mathrm{~min}$ at $37^{\circ} \mathrm{C}$ in complete medium to allow trafficking of transferrin from early endosomes to recycling endosomes. Flattened threedimensional images were captured for each treatment using identical conditions. The background intensity ROI was selected from a region not containing cells. Background intensity was removed from the entire field of view before individual cells were selected, and total cell transferrin intensity was then measured for analysis ( $n>30$ cells per animal).

Dextran uptake assay. RAW 264.7 macrophages were incubated with or without LPS pre-priming for $30 \mathrm{~min}$. Alexa Fluor 488-conjugated dextran $(10,000 \mathrm{MW})$ was added to the cells at a final concentration of $50 \mu \mathrm{g} \mathrm{ml}^{-1}$ in complete medium and left on cells for $10 \mathrm{~min}$. Macropinocytosis was stopped by washing cells in $4^{\circ} \mathrm{C}$ PBS before exchanging for $4^{\circ} \mathrm{C}$ complete medium. Cells were kept on ice until imaging live using the Personal DeltaVision deconvolution microscope. Macropinocytosis was then analysed using ImageJ software. Cells were segmented using WGA as a cell mask. Macropinosomes were segmented and filtered by size $>0.2 \mu \mathrm{m}$. Final segmentation of individual macropinosomes was performed manually before counting and size measurements were taken in an automated fashion.

Ruffle enrichment analysis. Recruitment analysis was performed by comparing the fluorescence intensity of a transfected protein at multiple locations in a given cell. The peak fluorescence intensity of each channel across an ROI line was divided by the fluorescent intensity of WGA as a measure of total membrane. The mean fluorescence of each channel/WGA at the filopodia was set to 1 and the measurements in ruffles compared with identify recruitment of tdTomato-Rab8a and GFP-Akt-PH. By standardizing all measurements to the WGA intensity at the filopodia, measurements among cells could be compared even though the total intensity per cell was not equal.

Microscopy. For live cell experiments, RAW 264.7 macrophages were cultured on glass-bottom 35-mm dishes (MatTek). Live and fixed cell imaging were additionally performed using a Personal DeltaVision Olympus IX71 inverted wide-field deconvolution microscope equipped with Olympus U-Apochromat $40 \times / 1.35$ oil DIC, Plan-Apochromat $60 \times / 1.42$ oil DIC and UPLS-Apochromat $100 \times / 1.40$ oil DIC, and a $120 \mathrm{~W}$ xenon arc lamp. Images were captured using a Roper Coolsnap HQ2 monochrome camera. Live cell three-dimensional $z$-stack imaging for ruffle analysis using temporal colour coding was acquired using the Zeiss Axiovert 200 Yokogawa spinning disk confocal microscope. Each stack was acquired every $30 \mathrm{~s}$ and full stacks represented as sum-intensity projections. Temporal colour coding of each slice was performed using ImageJ and the spectrum lookup table. Immunofluorescence staining is described in 'phagocytic assay and procedures' ${ }^{63,64}$.

Scanning electron microscopy. RAW 264.7 macrophage cells were incubated with human IgG-opsonized latex beads ( $3 \mu \mathrm{m}$, Sigma-Aldrich) for $30 \mathrm{~min}$ and fixed with $2.5 \%$ glutaraldehyde in sodium cacodylate buffer, post-fixed in $2 \%$ osmium tetroxide, dehydrated through ethanol, and dried using HMDS (Sigma-Aldrich). Coverslips were coated in platinum and viewed on a JEOL JSM-6300F scanning electron microscope.

Immunoblot. For immunoblot analysis ${ }^{65}$, cells were lysed in lysis buffer $(20 \mathrm{mM}$ HEPES pH 7.4, $100 \mathrm{mM} \mathrm{NaCl}, 1 \% \mathrm{NP}-40$ (Sigma) with addition of $1 \mathrm{mM}$ PMSF, $1 \mathrm{mM}$ DTT, cOmplete protease inhibitors (Roche Applied Science) and phosphoStop tablets (Roche Applied Science). The proteins were transferred to a polyvinylidene difluoride membrane (BioTrace, NZ), blocked with 5\% skim milk in PBS- $0.1 \%$ Tween- 20 buffer (TBS-T) and incubated overnight at $4{ }^{\circ} \mathrm{C}$ with primary antibodies. After washing of the membrane, a secondary antibody was incubated for 1 hour. The membrane was developed with a chemiluminescence reagent (ECL, Detection Reagents, Thermo Scientific) according to the manufacturer's instructions.

Quantitative real-time PCR and enzyme-linked immunosorbent assay. Total RNA was prepared using RNeasy mini-kits (Qiagen, Valencia, CA) and cDNA reverse transcribed using $1 \mu \mathrm{g}$ of total RNA and Superscript III reverse transcriptase (Invitrogen, Carlsbad, CA). Gene expression was quantitated using SYBR Green PCR master mix (Applied Biosystems) ${ }^{66}$, using an ABI Prism 7000 sequence detection system (Applied Biosystems, Foster City, CA). All primers used in this study are listed in Supplementary Table 2. Data are presented as relative change compared with control cells $(n=3)$ and represents the average \pm s.e.m.

Rab8a GTP loading for GST pull-down and mass spectrometry analysis. GTPloaded GST-Rab8a sepharose beads were incubated with LPS-activated cell lysate for $1 \mathrm{~h}$ at $4{ }^{\circ} \mathrm{C}$ with agitation. MicroSpin columns (\#27-3565-01; GE Healthcare) were used for all of the pull-downs. Beads were washed with ice-cold wash buffer (50 mM Tris, $150 \mathrm{mM} \mathrm{NaCl}$, 1\% NP-40, 1 mM PMSF, 1 mM DTT, pH 7.4). Elution was achieved conventionally by boiling in $3 \times$ SDS-PAGE sample buffer for $5 \mathrm{~min}$ (ref 67). LC MS/MS was performed at the IMB Mass Spectrometry Facility, The University of Queensland. LPS-stimulated RAW 264.7 macrophage extracts were lysed in ice-cold lysis buffer (20 mM HEPES pH 7.4, $150 \mathrm{mM} \mathrm{NaCl}, 1 \% \mathrm{NP}-40$ (Sigma), $1 \mathrm{mM}$ PMSF, $1 \mathrm{mM}$ DTT, cOmplete protease inhibitors (Roche Applied Science), and PhosStop tablet (Roche Applied Science). The lysate was centrifuged at $75,600 \mathrm{~g}$ for $15 \mathrm{~min}$ at $4{ }^{\circ} \mathrm{C}$. The supernatant was pre-cleared by the addition of GSH-Sepharose beads for $1 \mathrm{~h}$, pelleted at $50 \mathrm{~g}$ for $5 \mathrm{~min}$ at $4{ }^{\circ} \mathrm{C}$, and the supernatant collected. Various GST-tagged recombinant proteins were then incubated with an equal amount of tissue lysate at $4^{\circ} \mathrm{C}$ for $1 \mathrm{~h}$. Beads were washed extensively with ice-cold $20 \mathrm{mM}$ Tris $\mathrm{pH} 7.4$ containing $150 \mathrm{mM} \mathrm{NaCl}, 1 \mathrm{mM}$ DTT, and $1 \mathrm{mM}$ PMSF, eluted in $2 \times$ SDS-PAGE sample buffer, resolved on 10\% SDS-PAGE gels, and stained with Coomassie blue G250. GTP loading of Rab8 was performed by nucleotide exchange as described earlier ${ }^{68}$.

Samples were analysed by LC MS/MS on a Shimadzu Prominence Nano HPLC (Japan) coupled to a Triple TOF 5600 mass spectrometer (ABSCIEX, Canada) equipped with a nano electrospray ion source. Six microliters of each extract was injected onto a $50 \mathrm{~mm} \times 300 \mu \mathrm{m}$ C18 trap column (Agilent Technologies, Australia) at $30 \mu \mathrm{lmin}^{-1}$. The samples were de-salted on the trap column for $5 \mathrm{~min}$ using $0.1 \%$ formic acid at $30 \mu \mathrm{lmin}-1$. The trap column was then placed in-line with the analytical nano HPLC column, a $150 \mathrm{~mm} \times 75 \mu \mathrm{m} 300 \mathrm{SBC} 18$, $3.5 \mu \mathrm{m}$ (Agilent Technologies, Australia) for mass spectrometry analysis. Linear gradients of $1-40 \%$ solvent $B$ over $35 \mathrm{~min}$ at $300 \mathrm{nl} \mathrm{min}^{-1}$ flow rate, followed by a steeper gradient from 40 to $80 \%$ solvent B in 5 min were used for peptide elution. Solvent $\mathrm{B}$ was held at $80 \%$ for $5 \mathrm{~min}$ for washing the column, and then returned to $1 \%$ solvent $\mathrm{B}$ for equilibration before the next sample injection. Solvent $\mathrm{A}$ consisted of $0.1 \%$ formic acid and solvent $B$ contained $90 / 10$ acetonitrile $/ 0.1 \%$ formic acid. The ion spray voltage was set to $2400 \mathrm{~V}$, declustering potential (DP) $100 \mathrm{~V}$, curtain gas flow 25 , nebuliser gas 1 (GS1) 12 , and interface heater at $150^{\circ} \mathrm{C}$. The mass spectrometer acquired $500 \mathrm{~ms}$ full scan TOF-MS data followed by 20 by $50 \mathrm{~ms}$ full scan product ion data in an information dependent acquisition (IDA) mode. Full scan TOF-MS data were acquired over the mass range 350-1400 and for product ion $\mathrm{ms} / \mathrm{ms} 80-1400$. Ions observed in the TOF-MS scan exceeding a threshold of 100 counts and a charge state of +2 to +5 were set to trigger the acquisition of product ion, $\mathrm{ms} / \mathrm{ms}$ spectra of the resultant 20 most intense ions. The data were acquired and processed using Analyst TF 1.6.1 software (ABSCIEX, Canada). Proteins were identified by database searching usingProteinPilot v4.5 (ABSCIEX, Canada) against the UniProt_Sprot_20130205 database ( 106,000 entries of all species searched, FDR of 1\%). Search parameters were defined as a thorough search using trypsin digestion, iodoacetamide cysteine alkylation and all entries in the database. Proteins were considered identified if there was at least one peptide identified with $99 \%$ confidence.

Image analysis software. All images were analyzed using either ImageJ (version 1.43; National Institutes of Health, Maryland, USA) or Imaris (version 7.3.6, Bitplane Scientific Software). Adobe Photoshop CS6 was used to crop regions of interest or to extract single channels.

Statistics. If not stated otherwise, data are presented as arithmetic means \pm s.e.m. For direct comparison of one experimental variation, Student's $t$-test was used for data with normal distribution (assessed by Shapiro-Wilk test). Post-test analysis of one-way analysis of variance was corrected for multiple comparisons by Dunett's method and corrected for multiple comparisons in two-way analysis of variance using Sidak's method. All analysed experiments used biological replicates to compute statistical significance. In all statistical analysis, a $P$-value $<0.05$ was considered statistically significant. Statistics were calculated using GraphPad Prism version 7.0 (GraphPad Software, San Diego, CA).

\section{References}

1. Stow, J. L. \& Murray, R. Z. Intracellular trafficking and secretion of inflammatory cytokines. Cytokine Growth Factor Rev. 24, 227-239 (2013).

2. Zerial, M. \& McBride, H. Rab proteins as membrane organizers. Nat. Rev. Mol. Cell Biol. 2, 107-117 (2001).

3. Wang, D. et al. Ras-related protein Rab10 facilitates TLR4 signalling by promoting replenishment of TLR4 onto the plasma membrane. Proc. Natl Acad. Sci. USA 107, 13806-13811 (2010).

4. Wang, Y. Z. et al. Lysosome-associated small Rab GTPase Rab7b negatively regulates TLR4 signalling in macrophages by promoting lysosomal degradation of TLR4. Blood 110, 962-971 (2007). 
5. Husebye, H. et al. The Rab1la GTPase controls Toll-like receptor 4-induced activation of interferon regulatory factor-3 on phagosomes. Immunity 33, 583-596 (2010).

6. Smith, A. C. et al. A network of Rab GTPases controls phagosome maturation and is modulated by Salmonella enterica serovar Typhimurium. J. Cell Biol. 176, 263-268 (2007).

7. Seto, S., Tsujimura, K. \& Koide, Y. Rab GTPases regulating phagosome maturation are differentially recruited to mycobacterial phagosomes. Traffic 12, 407-420 (2011).

8. Rifkin, I. R., Leadbetter, E. A., Busconi, L., Viglianti, G. \& Marshak-Rothstein, A. Toll-like receptors, endogenous ligands, and systemic autoimmune disease. Immunol. Rev. 204, 27-42 (2005).

9. Akira, S. \& Takeda, K. Toll-like receptor signalling. Nat. Rev. Immunol. 4, 499-511 (2004).

10. Aksoy, E. et al. The p110 delta isoform of the kinase PI(3)K controls the subcellular compartmentalization of TLR4 signalling and protects from endotoxic shock. Nat. Immunol. 13, 1045-1054 (2012).

11. Zanoni, I. et al. CD14 controls the LPS-induced endocytosis of Toll-like receptor 4. Cell 147, 868-880 (2011).

12. Kagan, J. C. et al. TRAM couples endocytosis of Toll-like receptor 4 to the induction of interferon-beta. Nat. Immunol. 9, 361-368 (2008).

13. Kagan, J. C. \& Medzhitov, R. Phosphoinositide-mediated adaptor recruitment controls toll-like receptor signalling. Cell 125, 943-955 (2006).

14. O’Neill, L. A. J. \& Bowie, A. G. The family of five: TIR-domain-containing adaptors in Toll-like receptor signalling. Nat. Rev. Immunol. 7, 353-364 (2007).

15. Kawai, T. \& Akira, S. The role of pattern-recognition receptors in innate immunity: update on Toll-like receptors. Nat. Immunol. 11, 373-384 (2010)

16. Weichhart, T. et al. The TSC-mTOR signalling pathway regulates the innate inflammatory response. Immunity 29, 565-577 (2008).

17. Guertin, D. A. \& Sabatini, D. M. Defining the role of $\mathrm{mTOR}$ in cancer. Cancer Cell 12, 9-22 (2007).

18. Cook, D. N., Pisetsky, D. S. \& Schwartz, D. A. Toll-like receptors in the pathogenesis of human disease. Nat. Immunol. 5, 975-979 (2004).

19. Patel, P. C. \& Harrison, R. E. Membrane ruffles capture C3bi-opsonized particles in activated macrophages. Mol. Biol. Cell 19, 4628-4639 (2008).

20. Welliver, T. P., Chang, S. L., Linderman, J. J. \& Swanson, J. A. Ruffles limit diffusion in the plasma membrane during macropinosome formation. J. Cell Sci. 124, 4106-4114 (2011).

21. Abella, J. V., Parachoniak, C. A., Sangwan, V. \& Park, M. Dorsal ruffle microdomains potentiate met receptor tyrosine kinase signalling and downregulation. J. Biol. Chem. 285, 24956-24967 (2010).

22. Buccione, R., Orth, J. D. \& McNiven, M. A. Foot and mouth: podosomes, invadopodia and circular dorsal ruffles. Nat. Rev. Mol. Cell Biol. 5, 647-657 (2004).

23. Hattula, K. et al. Characterization of the Rab8-specific membrane traffic route linked to protrusion formation. J. Cell Sci. 119, 4866-4877 (2006).

24. Bohdanowicz, M. et al. Phosphatidic acid is required for the constitutive ruffling and macropinocytosis of phagocytes. Mol. Biol. Cell 24, 1700-1712 (2013).

25. Yoshida, S., Hoppe, A. D., Araki, N. \& Swanson, J. A. Sequential signalling in plasma-membrane domains during macropinosome formation in macrophages. J. Cell Sci. 122, 3250-3261 (2009).

26. Kavran, J. M. et al. Specificity and promiscuity in phosphoinositide binding by Pleckstrin homology domains. J. Biol. Chem. 273, 30497-30508 (1998).

27. Fukuda, M., Kanno, E., Ishibashi, K. \& Itoh, T. Large scale screening for novel rab effectors reveals unexpected broad Rab binding specificity. Mol. Cell. Proteomics 7, 1031-1042 (2008).

28. Hirsch, E. et al. Central role for G protein-coupled phosphoinositide 3-kinase gamma in inflammation. Science 287, 1049-1053 (2000).

29. Schmid, M. C. et al. Receptor tyrosine kinases and TLR/IL1 Rs unexpectedly activate myeloid cell PI3K gamma, a single convergent point promoting tumor inflammation and progression. Cancer Cell 19, 715-727 (2011).

30. Suire, S. et al. G beta gamma s and the Ras binding domain of p110 gamma are both important regulators of $\mathrm{PI}(3) \mathrm{K}$ gamma signalling in neutrophils. Nat. Cell Biol. 8, 1303-1309 (2006).

31. Suire, S. et al. p84, a new G beta gamma-activated regulatory subunit of the type IB phosphoinositide 3-kinase p110 gamma. Curr. Biol. 15, 566-570 (2005).

32. Brock, C. et al. Roles of G beta gamma in membrane recruitment and activation of p110 gamma/p101 phosphoinositide 3-kinase gamma. J. Cell Biol. 160, 89-99 (2003).

33. Hou, X. M. et al. A structural basis for Lowe syndrome caused by mutations in the Rab-binding domain of OCRL1. EMBO J. 30, 1659-1670 (2011).

34. Pacold, M. E. et al. Crystal structure and functional analysis of Ras binding to its effector phosphoinositide 3-kinase gamma. Cell 103, 931-943 (2000).

35. Camps, M. et al. Blockade of PI3Kgamma suppresses joint inflammation and damage in mouse models of rheumatoid arthritis. Nat. Med 11, 936-943 (2005).
36. Swanson, J. A. Shaping cups into phagosomes and macropinosomes. Nat. Rev. Mol. Cell Biol. 9, 639-649 (2008).

37. Jean, S. \& Kiger, A. A. Coordination between RAB GTPase and phosphoinositide regulation and functions. Nat. Rev. Mol. Cell Biol. 13, 463-470 (2012).

38. Katholnig, K., Linke, M., Pham, H., Hengstschlager, M. \& Weichhart, T. Immune responses of macrophages and dendritic cells regulated by mTOR signalling. Biochem. Soc. Trans. 41, 927-933 (2013).

39. Ivanov, S. S. \& Roy, C. R. Pathogen signatures activate a ubiquitination pathway that modulates the function of the metabolic checkpoint kinase mTOR. Nat. Immunol. 14, 1219-1228 (2013).

40. Peranen, J. Rab8 GTPase as a regulator of cell shape. Cytoskeleton 68, 527-539 (2011).

41. Knodler, A. et al. Coordination of Rab8 and Rab11 in primary ciliogenesis. Proc. Natl Acad. Sci. USA 107, 6346-6351 (2010).

42. Honda, A. et al. Phosphatidylinositol 4-phosphate 5-kinase alpha is a downstream effector of the small G protein ARF6 in membrane ruffle formation. Cell 99, 521-532 (1999).

43. Hokanson, D. E. \& Bretscher, A. P. EPI64 interacts with Slp1/JFC1 to coordinate Rab8a and Arf6 membrane trafficking. Mol. Biol. Cell 23, 701-715 (2012).

44. Christoforidis, S. et al. Phosphatidylinositol-3-OH kinases are Rab5 effectors. Nat. Cell Biol. 1, 249-252 (1999).

45. Vadas, O. et al. Molecular determinants of PI3Kgamma-mediated activation downstream of G-protein-coupled receptors (GPCRs). Proc. Natl Acad. Sci. USA 110, 18862-18867 (2013).

46. Fritsch, R. et al. RAS and RHO Families of GTPases Directly Regulate Distinct Phosphoinositide 3-Kinase Isoforms. Cell 153, 1050-1063 (2013).

47. Laffargue, M. et al. Phosphoinositide 3-kinase gamma is an essential amplifier of mast cell function. Immunity 16, 441-451 (2002).

48. Weichhart, T. \& Saemann, M. D. The multiple facets of mTOR in immunity. Trends Immunol. 30, 218-226 (2009).

49. Kim, J. H., Yoon, M. S. \& Chen, J. Signal transducer and activator of transcription 3 (STAT3) mediates amino acid inhibition of insulin signalling through Serine 727 phosphorylation. J. Biol. Chem. 284, 35425-35432 (2009).

50. Ruckle, T., Schwarz, M. K. \& Rommel, C. PI3K gamma inhibition: towards an 'aspirin of the 21 st century'? Nat. Rev. Drug Discovery 5, 903-918 (2006).

51. Kobayashi, N. et al. Blockade of class IB phosphoinositide-3 kinase ameliorates obesity-induced inflammation and insulin resistance. Proc. Natl Acad. Sci. USA 108, 5753-5758 (2011).

52. Wymann, M. P. \& Solinas, G. Inhibition of phosphoinositide 3-kinase gamma attenuates inflammation, obesity, and cardiovascular risk factors. Ann. NY Acad. Sci. 1280, 44-47 (2013).

53. Bakowski, M. A. et al. The phosphoinositide phosphatase SopB manipulates membrane surface charge and trafficking of the Salmonella-containing vacuole. Cell. Host. Microbe. 7, 453-462 (2010).

54. Heo, W. D. et al. $\mathrm{PI}(3,4,5) \mathrm{P}-3$ and $\mathrm{PI}(4,5) \mathrm{P}-2$ lipids target proteins with polybasic clusters to the plasma membrane. Science 314, 1458-1461 (2006).

55. Collins, B. M., Skinner, C. F., Watson, P. J., Seaman, M. N. J. \& Owen, D. J. Vps29 has a phosphoesterase fold that acts as a protein interaction scaffold for retromer assembly. Nat. Struct. Mol. Biol. 12, 594-602 (2005).

56. Tushinski, R. J. et al. Survival of mononuclear phagocytes depends on a lineagespecific growth-factor that the differentiated cells selectively destroy. Cell $\mathbf{2 8}$, 71-81 (1982).

57. Hume, D. A. \& Gordon, S. Regulation of Bone-Marrow Macrophage Proliferation. Adv. Exp. Med. Biol. 155, 261-266 (1982).

58. Shurety, W., Merino-Trigo, A., Brown, D., Hume, D. A. \& Stow, J. L. Localization and post-Golgi trafficking of tumor necrosis factor-alpha in macrophages. J. Interferon Cytokine Res. 20, 427-438 (2000).

59. Rubinson, D. A. et al. A lentivirus-based system to functionally silence genes in primary mammalian cells, stem cells and transgenic mice by RNA interference (vol 33, pg 401, 2003). Nat. Genet 39, 803-803 (2007).

60. Vitriol, E. A., Uetrecht, A. C., Shen, F. M., Jacobson, K. \& Bear, J. E. Enhanced EGFP-chromophore-assisted laser inactivation using deficient cells rescued with functional EGFP-fusion proteins. Proc. Natl Acad. Sci. USA 104, 6702-6707 (2007).

61. Rubinson, D. A. et al. A lentivirus-based system to functionally silence genes in primary mammalian cells, stem cells and transgenic mice by RNA interference. Nat. Genet. 33, 401-406 (2003).

62. Kutner, R. H., Zhang, X. Y. \& Reiser, J. Production, concentration and titration of pseudotyped HIV-1-based lentiviral vectors. Nat. Protoc. 4, 495-505 (2009).

63. Yeo, J. C., Wall, A. A., Stow, J. L. \& Hamilton, N. A. High-throughput quantification of early stages of phagocytosis. BioTechniques 55, 24-32 (2013). 
64. Pagan, J. K. et al. The t-SNARE syntaxin 4 is regulated during macrophage activation to function in membrane traffic and cytokine secretion. Curr. Biol. 13, 156-160 (2003).

65. Murray, R. Z., Wylie, F. G., Khromykh, T., Hume, D. A. \& Stow, J. L. Syntaxin 6 and Vtilb form a novel SNARE complex, which is up-regulated in activated macrophages to facilitate exocytosis of tumor necrosis Factor-alpha. J. Biol. Chem. 280, 10478-10483 (2005)

66. Schroder, K. et al. Conservation and divergence in Toll-like receptor 4-regulated gene expression in primary human versus mouse macrophages. Proc. Natl Acad. Sci. USA 109, E944-E953 (2012).

67. Luo, L., King, N. P., Yeo, C. J., Jones, A. \& Stow, J. L. Single-step protease cleavage elution for identification of protein-protein interactions from GST pull-down and mass spectrometry. Proteomics 14, 19-23 (2014).

68. Simon, I., Zerial, M. \& Goody, R. S. Kinetics of interaction of Rab5 and Rab7 with nucleotides and magnesium ions. J. Biol. Chem. 271, 20470-20478 (1996).

\section{Acknowledgements}

We thank Juliana Venturato, Tatiana Khromykh, Darren Brown and Imala Alwis for expert technical assistance and we thank colleagues as acknowledged for reagents. We also thank Fiona Wylie and Nathan King for critical reading of the manuscript. Imaging was performed in the Australian Cancer Research Foundation-funded Cancer Biology

Imaging Facility at IMB. This work was supported by funding from the National Health and Medical Research Council of Australia, including a funded programme (606788) J.L.S., E.L.H.; fellowship (1003021) J.L.S. and project (569543) J.L.S.

\section{Author contributions}

L.L. and A.A.W. contributed equally. L.L., A.A.W. and J.L.S. conceived the project, designed the experiments, analysed the data and wrote the manuscript. J.C.Y., N.D.C., K.W.C., S.J.N. and S.S. conducted experiments and analysis. B.M.C, E.L.H., B.J.J. K.S., S.J., and M.J.S. contributed data, reagents and advice.

\section{Additional information}

Supplementary Information accompanies this paper at http://www.nature.com/ naturecommunications

Competing financial interests: The authors declare no competing financial interests.

Reprints and permission information is available online at http://npg.nature.com/ reprintsandpermissions/

How to cite this article: Luo, L. et al. Rab8a interacts directly with PI3K $\gamma$ to modulate TLR4-driven PI3K and mTOR signalling. Nat. Commun. 5:4407 doi: 10.1038/ ncomms5407 (2014). 